TRAVAIL

ET EMPLOI

\section{Travail et Emploi}

153 | janvier-mars 2018

La Garantie jeunes : éléments d'évaluation et de comparaison internationale

\title{
La Garantie européenne pour la jeunesse
}

Bilan systématique des mises en œuvre dans les pays membres

The European Youth Guarantee. A systematic review of its implementation

across countries

Verónica Escudero et Elva López Mourelo

\section{(2) OpenEdition}

1 Journals

\section{Édition électronique}

URL : http://journals.openedition.org/travailemploi/7983

DOI : 10.4000/travailemploi.7983

ISSN : 1775-416X

Éditeur

DARES - Ministère du Travail

\section{Édition imprimée}

Date de publication : 1 janvier 2018

Pagination : 89-122

ISSN : 0224-4365

\section{Référence électronique}




\title{
La Garantie européenne pour la jeunesse
}

\author{
Bilan systématique des mises en œuvre \\ dans les pays membres"
}

\author{
Verónica Escudero ${ }^{* *}$, Elva López Mourelo ${ }^{* * *}$
}

\begin{abstract}
La Garantie européenne pour la jeunesse (ou Garantie jeunesse) apparaît comme une politique du marché du travail innovante. Elle a été promue dans un contexte où se faisait sentir le besoin d'une réponse urgente face aux conséquences durablement préjudiciables du chômage de longue durée. Cet article examine les données empiriques sur l'efficacité des expériences antérieures de mesures ciblées sur les jeunes, ainsi que les évaluations existantes de la Garantie jeunesse, afin d'identifier les facteurs qui s'avèrent décisifs pour sa réussite. Il détermine ensuite si ces facteurs clés de réussite sont intégrés dans les plans de mise en œuvre élaborés par les pays européens et regarde dans quelles mesures les objectifs fixés ont été atteints dans la pratique. La plupart des pays ont établi des critères d'éligibilité clairs ainsi que des cadres institutionnels appropriés et proposent un ensemble complet de mesures ; cependant, bon nombre d'entre eux ont encore des difficultés à atteindre les objectifs fixés en termes d'intervention précoce et à mettre en place des mécanismes d'exécution efficaces. Enfin, bien que les sommes effectivement investies par les États dans les programmes nationaux de Garantie jeunesse aient souvent dépassé les dépenses initialement prévues, les allocations de ressources se sont souvent avérées insuffisantes par rapport aux recommandations.
\end{abstract} $\coprod_{\text {a Garantie européenne pour la jeunesse (ou Garantie jeunesse, GJ) est l'une des }}$

\footnotetext{
* Les auteures tiennent à remercier Eva van Belle et Louise Nennen pour leur assistance de grande qualité dans la recherche, ainsi que Steven Tobin et les participants à la conférence organisée par le CEET «La Garantie jeunes et son évaluation » pour leurs utiles commentaires. Les opinions exprimées dans cet article et les conclusions présentées proviennent des auteures et ne représentent pas nécessairement celles de l'OIT.

** Organisation internationale du Travail, Département de la recherche, École d'économie de Paris - Paris School of Economics, EHESS ; escudero@ilo.org.

*** Organisation internationale du Travail, Département de la recherche ; lopezmourelo@ilo.org.
} 
Remarquable par sa conception, elle illustre également l'ampleur de l'engagement consenti par toutes les parties prenantes pour parvenir à des accords capables de soutenir sa mise en place. Aujourd'hui, la GJ européenne engage les États membres à garantir à tous les jeunes de moins de 25 ans ${ }^{1}$, dans les quatre mois suivant la perte de leur emploi ou leur sortie du système éducatif, une offre d'emploi de qualité et en adéquation avec leurs compétences ou leur expérience, ou bien la possibilité de suivre une formation ou d'entrer en apprentissage ou en stage professionnel (EUROPEAN COUNCIL, 2013). Par conséquent, dans sa conception, la GJ combine le concept de « garantie », qui s'accompagne d'un délai maximal pour que les pays prennent certaines mesures, et l'idée selon laquelle les mesures d'activation doivent, pour être efficaces, être de nature globale. En effet, la notion de « garantie » dans les programmes de GJ est fondée sur la mise en avant de droits, à la différence des politiques traditionnelles, plutôt fondées sur une vision utilitariste centrée sur l'obtention d'effets sur l'emploi. Par ailleurs, la GJ inclut une grande variété de mesures : des programmes de formation scolaire et professionnelle; des mesures de remédiation face au décrochage scolaire ; des services d'intermédiation vis-à-vis du marché du travail ; des politiques actives du marché du travail (PAMT, voir annexe 1 pour les abréviations) visant à agir sur la demande de travail, comme la création directe d'emplois, les subventions à l'embauche ou les incitations au démarrage d'entreprises. Tous ces facteurs, qui caractérisent la GJ et la distinguent des autres politiques de l'emploi, pourraient avoir des effets spécifiques encore inédits.

La GJ a été promue dans un contexte où il fallait, de façon urgente et importante, apporter une réponse à la situation des jeunes : dans l'Union européenne à 28 (UE-28), en 2013, le taux de chômage des jeunes de 15 à 24 ans atteignait 23,6 \% et dépassait les $50 \%$ dans certains pays comme la Grèce $(58,3 \%)$ et l'Espagne $(55,5 \%)$. De plus, on comptait un nombre très important de jeunes ne se trouvant ni en situation d'emploi, ni d'études, ni de formation (Not in Education, Employment, Training: Neet). En moyenne, en 2013, $13 \%$ des jeunes dans l'UE-28 étaient en situation de Neet, cette proportion pouvant atteindre plus de $20 \%$ dans le cas de la Bulgarie, de la Grèce et de l'Italie (voir annexe 2). Cette situation menaçait de retarder la reprise économique et de mettre en danger le modèle social européen. Elle entraînait également des conséquences potentiellement durables sur les trajectoires d'emploi des jeunes, avec des pertes durables en termes de salaires, une érosion des compétences et un risque accru de découragement et d'inactivité. La réponse apportée a cherché à faire face aux défis créés par cette situation : la GJ est en effet l'un des rares efforts soutenus aussi largement par toutes les parties prenantes, y compris les gouvernements, les partenaires sociaux et la société civile. En réalité - comme nous allons le montrer-la participation

1. L'âge minimum des bénéficiaires de la GJ diffère selon les pays et dépend de l'âge auquel s'achève la scolarisation obligatoire. En Autriche, par exemple, les initiatives visant à assurer une intervention et une activation précoces commencent dès les deux dernières années de scolarisation obligatoire (N.B. : la scolarisation est obligatoire jusqu'à 15 ans) et s'accompagnent d'activités liées à l'encadrement des jeunes. 
de tous les partenaires sociaux, dans la conception et la mise en œuvre de ces mesures, s'est avérée décisive pour assurer la mise en œuvre du dispositif.

La mise en œuvre de la GJ au niveau national a toutefois rencontré des limites et ne s'est pas faite sans heurts. En effet, les garanties jeunesse ne sont pas de simples ajustements apportés aux politiques de l'emploi déjà en place. Pour les instaurer, il a souvent été nécessaire de créer ou de réformer les dispositifs de formation professionnelle, les systèmes d'éducation et les services publics de l'emploi (SPE). De plus, la réussite de ces programmes repose sur leur capacité à produire des accords de coopération avec les organisations patronales, les syndicats, les écoles et centres de formation ainsi qu'avec les organisations non gouvernementales, accords dont la conclusion peut prendre du temps. Enfin, leur mise en œuvre a eu un coût important, estimé à 45 milliards d'euros (PPA, Parité de pouvoir d'achat) pour l'UE-28 en 2014 (ESCUDERO, LóPEZ MOURELO, $2015)^{2}$. Cette somme doit être considérée comme un investissement, au regard de la réduction significative des coûts associés au chômage des jeunes qu'un usage efficace du dispositif peut produire. Et malgré le soutien financier de l'Initiative pour l'emploi des jeunes (IEJ), un important effort financier au niveau national était nécessaire pour atteindre le niveau recommandé.

Dans ce contexte, notre article propose d'examiner les premiers résultats de la mise en œuvre des programmes européens de GJ au niveau national, afin d'estimer si les efforts consentis sont en passe d'atteindre les objectifs. Dans cette perspective, nous identifions six facteurs décisifs de réussite, à partir des expériences antérieures de garanties jeunesse et des évaluations existantes de programmes nationaux. Nous présentons ensuite l'état actuel de la mise en œuvre des programmes nationaux de GJ - en observant plus particulièrement la façon dont ces six facteurs de réussite sont pris en compte. Cet exercice nous permet de mettre en évidence les résultats et les leçons à tirer de la mise en œuvre de la GJ jusqu'à aujourd'hui. Il permet aussi de repérer certaines des difficultés qui subsistent.

\section{La Garantie jeunesse : de l'expérience scandinave à l'initiative européenne ${ }^{3}$}

Les garanties jeunesse ont émergé au cours des années 1980 et 1990 dans les pays scandinaves, pionniers dans la mise en œuvre de politiques actives du marché du travail. Ainsi, la Suède a initié la première garantie jeunesse en 1984, la Norvège a pris une initiative comparable en 1993 et le Danemark ainsi que la Finlande ont fait

2. Des analyses antérieures, produites par l'OIT, estimaient son coût à environ 21 milliards d'euros par an, notamment à partir de l'exemple suédois (ILO, 2012). Ces calculs correspondaient uniquement aux pays de l'Eurozone et prenaient en compte les jeunes sans emploi plutôt que le nombre de jeunes Neets.

3. Cette section s'appuie sur une recherche menée dans le cadre d'un article précédent sur le sujet (EsCUDERO, LÓPEZ Mourelo, 2015). 
de même en 1996 (MASCHERINI, 2012). En dehors de la région scandinave, le New Deal for Young People britannique (« Nouveau pacte pour les jeunes»), lancé en 1998, participe du même type d'intervention. Plus récemment, d'autres pays ont mis en place des programmes similaires, comme la Ausbildungsgarantie ( Garantie de formation ») autrichienne ou le Jeugdwerkplan («Plan de travail pour les jeunes ») néerlandais, lancés respectivement en 2008 et en 2007. Ces premières expériences de garantie jeunesse avaient pour point commun d'offrir une vaste gamme de mesures d'activation, qui pouvaient se combiner de différentes manières afin de s'adapter aux besoins particuliers des jeunes participants. Autre point de convergence, ces initiatives pionnières partageaient un même principe d'universalité et visaient tous les jeunes de moins de 25 ans, à l'exception du programme danois, où l'éligibilité était étendue jusqu'à 30 ans. Ces premières initiatives présentaient toutefois des divergences dans leurs objectifs : les garanties jeunesse mises en œuvre en Finlande, en Norvège et en Suède visaient particulièrement à améliorer les trajectoires scolaires des participants, tandis que l'accent était plutôt mis sur l'apprentissage dans le cas des programmes autrichien et danois (OECD, 2015).

La mise en œuvre de ces premières expériences convergeait sur un certain nombre de points. Tout d'abord, toutes insistaient sur l'élaboration de plans adaptés sur mesure aux besoins des jeunes dépourvus d'emploi et de formation. Deuxièmement, les SPE ont joué un rôle central dans la mise en œuvre d'une approche personnalisée. Enfin, le principe fondamental de ces programmes était, déjà, de garantir aux jeunes chômeurs un emploi, un programme d'études ou une formation professionnelle. Bien qu'ayant été modifiées au cours des dernières décennies, ces premières garanties jeunesse ont réduit de manière effective le chômage des jeunes, même durant la crise des années 1990, qui les avait mises à l'épreuve très peu de temps après leur création (ILO, 2012).

Les institutions de l'Union européenne ont commencé à prendre des mesures afin d'établir une garantie jeunesse européenne durant la seconde moitié des années 2000 (voir annexe 3). En 2005, alors que se préparaient les directives pour les politiques de l'emploi des États membres pour la période 2005-2008, le Conseil a affirmé que celles-ci devaient garantir que « chaque jeune chômeur se voie proposer un nouveau départ avant qu'il n'atteigne six mois de chômage ${ }^{4} »$. Par la suite, en 2008, durant la préparation des directives pour 2008-2010, le Conseil a décidé de réduire ce délai en offrant cette garantie dans les quatre mois suivant la fin de la scolarité des jeunes ${ }^{5}$ (EC, 2012b).

En 2010 cependant, alors que le taux de chômage des jeunes dans l'Union européenne atteignait un niveau sans précédent (à plus de $20 \%$ ), les efforts menés à l'échelle européenne révélaient leurs insuffisances. De multiples initiatives institutionnelles furent prises tout au long de l'année 2010, dont des appels, lancés par le Parlement, la Commission européenne (CE) et le Forum européen de la jeunesse, pour

4. Décision 2005/600/CE du 12 juillet 2005, JO L 205, 6.8.2005, p. 21.

5. Décision 2008/618/CE du 15 juillet 2008, JO L 198, 26.7.2008, p. 47. 
l'établissement d'une garantie jeunesse européenne. En septembre 2010, la CE a lancé l'initiative « Jeunesse en mouvement » proposant un ensemble de mesures susceptibles de réduire le chômage des jeunes. Dans la communication relative à cette initiative, la $\mathrm{CE}$ a pour la première fois mis en avant le concept qui sous-tend aujourd'hui la GJ : assurer que tous les jeunes chômeurs se voient proposer une offre d'emploi ou une formation dans les quatre mois suivant la fin de leur scolarité (EC, 2010). Des appels similaires se sont répétés en 2011, bien que, dans la pratique, ils n'aient pas abouti au résultat escompté (EC, 2012c).

Des mesures plus concrètes ont été prises en 2012. En avril de cette année-là, la $\mathrm{CE}$ a promu un ensemble de mesures pour l'emploi dans sa communication intitulée « Vers une reprise génératrice d'emplois », incluant la proposition d'adopter en fin d'année une recommandation du Conseil afin de lancer une garantie jeunesse à l'échelle européenne (EC, 2012a). Un mois plus tard, le Parlement européen a répété l'importance d'instaurer un programme de garantie jeunesse, en insistant sur le fait que cette garantie jeunesse devait être légalement contraignante (EC, 2012c). Dans le même esprit, dans ses conclusions du 29 juin 2012, le Conseil européen affirmait que de telles mesures pourraient recevoir l'appui financier du Fonds social européen et que les États membres pourraient financer des subventions à l'emploi à travers ce Fonds. Enfin, en décembre 2012, la CE a lancé une série de mesures pour l'emploi des jeunes, incluant la proposition d'une recommandation du Conseil sur l'établissement d'une garantie jeunesse (EC, 2012b). Cette proposition a instauré les principaux éléments de la $\mathrm{GJ}^{6}$ et précisé les mécanismes par lesquels la CE pouvait soutenir sa mise en œuvre dans les États membres, notamment le soutien financier, le partage des bonnes pratiques et le suivi des mesures adoptées (EC, 2012b). Par la suite, en avril 2013, le Conseil a adopté cette proposition en tant que recommandation aux États membres (EUROPEAN COUNCIL, 2013). Il est important de souligner que la mise en place de la GJ européenne a en permanence bénéficié du soutien des partenaires sociaux, qui ont aussi joué un rôle actif durant tout le processus des négociations (BUSSI, GEYER, 2013) ${ }^{7}$.

Tout au long de l'année 2013, ont commencé les négociations en vue du financement du programme de la GJ. Dans la première moitié de l'année, le Conseil européen a créé l'IEJ (Initiative pour l'emploi des jeunes), avec une dotation de 6,4 milliards d'euros, pour soutenir la mise en œuvre de la GJ sur la période 2014-2016. Ce fonds était uniquement destiné aux régions où le taux de chômage avait dépassé les $25 \%$ en 2012 ; le soutien financier devait être octroyé à condition que les pays contrebalancent cette subvention par une contribution d'un montant au moins équivalent, extraite de

6. Ces principes comportaient : 1) l'interaction entre toutes les parties prenantes ; 2) l'intervention et l'activation précoces ; 3) le soutien à l'intégration dans le marché du travail ; 4) l'utilisation des Fonds structurels européens ; 5) le suivi et l'évaluation.

7. Une série de discussions entre les gouvernements et les partenaires sociaux a eu lieu entre avril et octobre 2012, concernant la mise en œuvre de la GJ et les facteurs qui seraient déterminants pour sa réussite. 
l'allocation reçue du Fonds social européen ${ }^{8}$. De plus, le Conseil européen incita les États membres à présenter leurs plans de mise en œuvre de la GJ avant la fin de l'année 2013, dans l'objectif de les promulguer en 2014 (EUROPEAN CounCIL, 2013, EC, 2013). En mai 2014, tous les États membres avaient soumis leurs plans de mise en œuvre, qui détaillaient les actions à mener pour l'établissement de la GJ, le rôle des principales institutions impliquées, un calendrier de mise en œuvre et un schéma de financement des différentes initiatives. Bien que la mise en œuvre ait commencé en 2014, la plupart des mesures ont été déployées progressivement et, comme nous le détaillons plus bas, certaines restent encore à exécuter. Étant donné l'ampleur des besoins, la CE a proposé d'augmenter le budget de l'IEJ de 2,2 milliards d'euros pour la période 2017-20209 (EC, 2016d). Comme durant la période 2014-2016, l'allocation de l'IEJ doit être complétée par les États membres sur leurs propres ressources financières.

\section{Quels facteurs de réussite des plans nationaux de Garantie jeunesse ?}

\section{Quelques enseignements de la littérature sur l'efficacité des mesures de politique active de l'emploi}

Les propositions qui portent les plans de garantie jeunesse renvoient à trois composantes distinctes, mais complémentaires, des politiques publiques : les effets potentiels des PAMT, ceux du système scolaire et, enfin, ceux de l'approche basée sur les droits. Comme l'indique la littérature économique, les PAMT sont des mécanismes indispensables pour réduire les déséquilibres entre l'offre et la demande de main-d'œuvre ; accroître la productivité de la main-d'œuvre ; faire en sorte que les chômeurs de longue durée et les autres groupes vulnérables gardent le contact avec le marché du travail (LAYARD et al., 2009 ; EsCUDERO, 2015). Ainsi, dans la mesure où les plans nationaux de GJ s'appuient sur un ensemble de PAMT, l'incidence prévisible du dispositif sera fonction des types de mesures mises en œuvre dans chaque pays, ainsi que de l'efficacité potentielle de chaque intervention.

Il existe une importante littérature concernant les effets économiques de chaque catégorie de PAMT recommandée dans le cadre des plans de GJ. Plus spécifiquement, le volet « formation » des programmes de garantie jeunesse est censé avoir une incidence significative sur le chômage des jeunes, bien que son incidence sur le chômage global puisse être limitée (SCHMID, 1996). En effet, ces mesures sont d'ordinaire efficaces

8. Parmi les 28 pays de l'UE, 20 étaient considérés comme éligibles au financement par l'IEJ. L'Autriche, le Danemark, l'Estonie, la Finlande, l'Allemagne, le Luxembourg, Malte et les Pays-Bas n'étaient pas considérés comme éligibles, parce qu' aucune région, à l'intérieur de leurs frontières, ne dépassait le seuil limite du taux de chômage des jeunes (ce qui ne les a pas empêchés de mettre en œuvre des programmes de GJ).

9. En juin 2017, cette proposition n'avait pas encore été adoptée par le Conseil européen, ni par le Parlement européen. 
pour réduire la vulnérabilité des groupes les plus exposés sur le marché du travail, comme les jeunes, ce qui pourrait mener à un effet de substitution réduisant l'emploi d'autres groupes sociaux (EsCUDERO, 2015). De plus, les programmes de formation peuvent induire un effet de verrouillage si les participants réduisent l'intensité de leur recherche d'emploi (BELLMANN, JACKMAN, 1996) ou un effet d'aubaine si on considère que l'embauche se serait produite même en l'absence de programme (CALMFORS, SKEDINGER, 1995).

De même, on peut s'attendre à ce que les services d'intermédiation et d'aide à la recherche d'emploi produisent des effets mitigés. D'un côté, ils peuvent accroître l'efficacité de la recherche d'emploi (SCHMid et al., 2001 ; BELlMANN, JACKMAN, 1996) et augmenter la demande de main-d'œuvre, puisqu'il devient moins coûteux d'ouvrir de nouveaux postes (PISSARIDES, 1990 ; CALMFORS, LANG, 1995). Mais d'un autre côté, ce type d'aide peut atténuer la crainte du chômage et donc réduire les efforts de recherche d'emploi (CALMFORS, SkEDINGER, 1995). Enfin, on s'attend à ce que les mesures conçues pour augmenter la demande de travail ciblée sur les jeunes, et qui font aussi partie des plans nationaux de GJ, augmentent les taux d'emploi, de façon directe (comme les recrutements dans le secteur public ou les subventions à l'embauche) ou de façon indirecte (comme les mesures visant à promouvoir la création d'entreprises) (EC, 2003). Cependant, ces mesures peuvent aussi produire les effets de déplacement et de substitution mentionnés plus haut (OECD, 1993 ; CALMFORS, SKEDINGER, 1995).

On peut également s'attendre à ce que les mesures qui concernent l'éducation dans les programmes de garantie jeunesse, jouent un rôle déterminant dans l'accès au marché du travail. D'après la théorie du capital humain, les individus dotés d'un capital plus élevé (notamment quand ils sont mieux instruits ou forts d'une plus grande expérience professionnelle) mènent une recherche d'emploi plus fructueuse (MiNCER, 1974). Les mécanismes conduisant à cet effet sont nombreux et divers. Tout d'abord, les candidats les mieux instruits sont censés s'adapter plus facilement à de nouvelles tâches (ThUROw, 1975) et mieux résister lorsque les conditions changent (SCHULTZ, 1975). En outre, il existe un effet de signal associé au niveau d'études, bien que tous les économistes ne s'accordent pas sur l'importance de cet effet. Alors que certains considèrent que l'effet signal des diplômes est déterminant (EDGERTON et al., 2012), d'autres estiment que comme l'éducation formelle n'est pas transférable directement en termes de compétences recherchées par les entreprises, cet effet n'est pas si important (BECKER, 1994 ; CARMECI, MAURO, 2003). Il y a toutefois consensus sur l'hypothèse selon laquelle l'effet de l'éducation sur l'emploi est fonction à la fois du type d'études suivies et du niveau de diplôme atteint (BECKER, 1994).

Enfin, l'efficacité des programmes de garantie jeunesse - comme celle des autres programmes mettant en avant le principe des droits (étant donné son statut de « garantie ») - dépend, au moins à court terme, de la capacité à faire correspondre la demande de main-d'œuvre aux engagements pris par les gouvernements (RAVALLION, 1991). En supposant que ce soit le cas et que l'économie puisse absorber les jeunes ainsi « assistés », les programmes basés sur le principe des droits devraient avoir un effet 
positif sur l'emploi en réduisant les pressions salariales, en stimulant l'économie et en amenant finalement à une augmentation du nombre d'emplois (DrÈZE, SEN, 1989 ; DEV, 1995 ; LAYARD, 1996). Cependant, si l'accroissement de la demande de main-d'œuvre est insuffisant, la garantie pour l'emploi, couplée au développement de la formation, implique que certains jeunes obtiendront des emplois à la place d'autres demandeurs d'emploi. Cette substitution en termes d'emplois n'est toutefois pas forcément un problème à long terme. En effet, dans la mesure où les demandeurs d'emploi évincés à court terme, en général plus immédiatement employables, trouveront un emploi plus facilement à mesure que la croissance économique s'accentuera, l'économie finira par bénéficier d'un taux de chômage global plus bas (LAYARD, 1996).

L'effet global des garanties jeunesse dépend en définitive grandement de la façon dont ces programmes sont conçus et mis en œuvre, tout comme du contexte dans lequel ils sont instaurés. Par conséquent, certains des effets négatifs associés à divers aspects des garanties jeunesse peuvent être atténués par les facteurs facilitant la réussite de ces interventions, comme l'indique la section suivante.

\section{Les facteurs clés de la réussite d'une mesure de type garantie jeunesse}

En raison du caractère récent de leur mise en œuvre, l'impact des programmes nationaux de GJ en Europe reste encore à évaluer de manière systématique. Certaines évaluations sur l'impact de programmes pilotes ont toutefois été lancées dans les trois dernières années. Massimiliano BRATTI et ses co-auteur.e.s (2017) évaluent par exemple l'impact d'un programme de formation professionnelle mis en œuvre en Lettonie en 2014, dans le cadre de l'ensemble de mesures lié à la GJ : aucun effet significatif en termes d'emploi n'est attribué à cette intervention. Des effets plus positifs sont observés dans une étude sur les conséquences de la mise en œuvre de la GJ dans des zones pilotes en France (DARES, 2016). L'étude, menée par la Dares (Direction de l'animation de la recherche, des études et de la statistique) relevant du ministère du Travail, utilise des données issues d'une enquête effectuée auprès de jeunes éligibles, dans des zones pilotes et des zones témoins, afin d'évaluer l'impact de la GJ sur le taux d'emploi des bénéficiaires. Les auteurs concluent que c'est dans les trois premiers mois ( si on considère la période de participation au programme ${ }^{10}$ ) et juste après la sortie du programme (pour l'après-participation) que le dispositif a l'effet le plus positif sur le taux d'emploi. Une approche différente, basée sur l'analyse sociologique qualitative ${ }^{11}$, souligne une certaine hétérogénéité derrière les effets globalement positifs associés au programme pilote mis en œuvre en France (LOISON-LERUSTE et al., 2016). En particulier, les auteurs concluent que, durant les premières années de mise en œuvre,

10. L'effet serait donc élevé pendant la participation au programme, ce qui est possible parce que les auteurs prennent en compte tous les types d'emploi, dont les stages, l'apprentissage, le service civique, les contrats subventionnés et les contrats standards.

11. Cette analyse est basée sur plus d'une centaine d'entretiens menés avec des jeunes ciblés par le programme et avec des conseillers des SPE chargés d'aider les participants. 
les trajectoires des bénéficiaires français sur le marché du travail se répartissent en trois catégories : un premier groupe de bénéficiaires a effectivement trouvé un emploi ; un deuxième groupe a continué sa préparation à l'emploi ; un dernier groupe, constitué de jeunes défavorisés, n'a pas pu tirer profit du programme en raison de la vulnérabilité de ce public et est demeuré dans une situation d'exclusion.

Les évaluations d'impact menées dans les pays pionniers des garanties jeunesse peuvent aussi apporter un éclairage sur l'efficacité de ces mesures pour faciliter la transition des jeunes vers le marché du travail (ESCUDERO et al., 2017). Des programmes pour la jeunesse, du type de ceux qui ont été mis en œuvre au Danemark, en Norvège et en Angleterre ${ }^{12}$, ont ainsi obtenu de très bons résultats en termes d'emploi et d'activation, à court et moyen terme (JENSEN et al., 2003 ; BLUNDELl et al., 2004 ; DE GIORGI, 2005 ; HARDOY, 2005). Dans la même veine, des analyses portant sur le cas suédois révèlent que les plans de garantie jeunesse peuvent être un moyen de faire face aux difficultés que le marché du travail pose aux jeunes, avec des résultats significatifs pour un coût relativement modéré (ILO, 2012). La garantie jeunesse lancée en Finlande en 2005 a aussi produit des effets positifs, mais seulement sur les chômeurs qualifiés (HÄMÄLÄINEN $e t$ $a l .$, 2017). De même, on observe des résultats mitigés dans le cas du plan flamand pour l'emploi des jeunes, établi en 2007, qui aurait légèrement augmenté les chances de trouver un emploi, mais au prix d'une diminution des salaires et du nombre de jours de travail (COCKX, VAN BELLE, 2016). Cet effet négatif serait même plus important chez les jeunes vivant dans des foyers au revenu modeste. Il est intéressant de constater que les effets les plus bénéfiques semblent être produits par les programmes les plus complets (ceux qui incluent un vaste ensemble de mesures différentes).

Outre les évaluations existantes, la plupart des éléments recommandés par la GJ européenne ont été largement étudiés, ce qui fournit des indications sur leurs résultats potentiels. On constate habituellement que ce sont les mesures concernant la formation scolaire et professionnelle qui sont les plus efficaces, mais principalement à moyen ou à long terme, au moment où les rendements de l'investissement en termes de capital humain tendent à être les plus élevés, alors qu'à court terme, les effets sont souvent négligeables (CARD et al., 2010). Les analyses empiriques mettent par ailleurs en évidence les effets positifs des mesures d'incitation à la création d'entreprise, qui incluent un « double dividende », dans la mesure où les entreprises subventionnées qui réussissent peuvent créer des emplois par la suite (CALIENDO, KüNn, 2014). De plus, les données actuelles suggèrent que l'aide à la recherche d'emploi et le suivi personnalisé des plans de carrière ont des effets positifs sur l'emploi (DOLTON, O'NEILL, 1996 ; VAN DEN BERG, VAN DER KLAAUW, 2006 ; MiCKLEWRIGHT, NAGY, 2010), à la fois à court et à moyen terme (CARD et al., 2017). Enfin, le fait de conditionner les prestations à la démonstration d'une recherche d'emploi effective promeut l'activation et aurait tendance à augmenter l'accès à l'emploi (GraVERSEN, vaN OURS, 2008 ; VAN DEN BERG et al., 2009).

12. Le programme New Deal for Young People (« Nouveau pacte pour les jeunes ») en Angleterre est très semblable aux garanties scandinaves. 
Ces analyses micro-économétriques, de même que les analyses macroéconomiques existantes (ESCUDERO et al., 2017 ; EsCUDERO 2015), soulignent le rôle fondamental de la conception et de la mise en ouvre des politiques pour la réussite des mesures d'activation. Sur la base des travaux de recherche et d'évaluation existants, il est ainsi possible de distinguer six prérequis pour que les programmes européens de GJ fonctionnent avec succès ${ }^{13}$, prérequis que nous détaillons dans les paragraphes qui suivent.

Premièrement, les critères d'éligibilité des dispositifs doivent être clairs. Ils doivent permettre d'identifier des sous-catégories à l'intérieur de la catégorie ciblée, afin de développer un ensemble de mesures adaptées aux besoins spécifiques de chaque catégorie. Les données provenant de la France (LOISON-LERUSTE et al., 2016) montrent par exemple que la situation de certains bénéficaires ne change pratiquement pas suite à leur entrée dans le dispositif. Il s'agit de jeunes en grande difficulté, qui ont besoin d'un soutien social et psychologique supplémentaire pour lever les barrières à l'accès à l'emploi, et pour qui des mesures uniquement destinées à accroître l'employabilité peuvent être insuffisantes. En l'occurrence, dans leur cas, les interventions doivent être personnalisées pour être les plus efficaces possible - ainsi, les mesures de formation scolaire et professionnelle doivent correspondre au niveau de qualification des jeunes. En effet, comme le suggèrent Marie LOISON-LERUSTE et ses co-auteur.e.s (2016), l'absence d'adéquation entre, d'une part, la formation fournie dans le cadre de la GJ et, d'autre part, la situation sociale et les besoins de qualification des participants, risque de les désengager plutôt que de renforcer leur implication dans le programme.

Deuxièmement, l'intervention précoce (dans les premiers mois de chômage) est souvent citée comme facteur de réussite. À titre d'exemple, une analyse de la garantie jeunesse finnoise de 2005, qui ne trouve d'efficacité que sur les jeunes les plus qualifiés, avance que l'absence d'impact sur les jeunes avec un faible niveau d'études s'explique par le fait que l'intervention précoce était déjà instituée à l'égard de ces derniers, de telle sorte que les nouvelles vagues d'activation (à la suite de la réforme de $2005^{14}$ ) n'ont affecté que les demandeurs d'emploi hautement qualifiés (HÄMÄLÄINEN et al., 2017). La nécessité de mettre en œuvre les garanties jeunesse dans un délai limité a été largement reconnue, puisqu'il est établi que des périodes prolongées de chômage produisent l'érosion des compétences et réduisent les efforts dans la recherche d'emploi. De fait, l'établissement d'un délai maximal de quatre mois, qui est l'une des caractéristiques de la GJ européenne, manifeste cette prise de conscience.

13. Nous avons déjà évoqué quelques-uns des prérequis pour que la GJ fonctionne avec succès : ESCUDERO, LÓPEZ MouRelo (2015). Nous reprenons, actualisons et étendons ici ces considérations, à partir de nouvelles études désormais disponibles sur l'efficacité de la GJ.

14. La réforme de 2005 concernant le programme de garantie jeunesse en Finlande a modifié les modalités des services destinés aux jeunes demandeurs d'emploi (17-24 ans) dans trois directions : 1) elle a introduit un entretien de conseil dans le mois suivant l'inscription ; 2) elle a fait passer de cinq à trois mois le délai maximal pour l'accomplissement du plan individuel de recherche d'emploi ; 3) elle a inclus des mesures d'activation dans le cadre de l'intervention (HÄMÄLÄINEN et al., 2017). 
En troisième lieu, les évaluations montrent que les mesures d'activation sont plus efficaces quand elles sont mises en œuvre sous la forme d'un ensemble complet de politiques, plutôt que par le biais de mesures isolées (ILO, 2016). Dans le cadre du programme britannique New Deal for Young People, par exemple, on a observé que la combinaison de différentes mesures d'activation augmentait la probabilité d'accès à l'emploi de près de cinq points de pourcentage (DE Giorgi, 2005). Parallèlement, les données indiquent que même au sein des programmes de formation, une combinaison d'éducation formelle de haute qualité et de formation sur le tas est nécessaire pour permettre aux jeunes de s'intégrer au marché du travail. À titre d'exemple, en Norvège, ce type de programmes complets en faveur de l'emploi accroît l'emploi à plein temps des femmes et des jeunes, tandis que les programmes de formation isolés n'ont pas d'effet significativement positif (HARDOY, 2005).

Le quatrième prérequis porte sur la création de cadres institutionnels appropriés, cruciale pour la réussite des programmes. À cet égard, il est important de souligner le rôle fondamental tenu par les SPE. L'évaluation de la période pilote du programme du New Deal conclut ainsi qu'au moins un cinquième de l'effet positif du programme sur l'emploi résulte de l'aide à la recherche et du soutien individualisé que fournit le bureau du SPE (BLuNDELl et al., 2004). En outre, de nombreux résultats soulignent l'importance des moyens en personnel dans les SPE (à la fois en termes d'effectifs et de compétences), capable d'offrir un soutien personnalisé à différents groupes et de gérer toute la gamme de services offerts sous l'égide des programmes de GJ. Dans le contexte des récents programmes de GJ mis en œuvre en France, par exemple, la Dares (2016) avance que les effets les plus positifs repérables dans les trois premiers mois de participation au programme peuvent être attribués au soutien très intense des conseillers durant cette première phase. Dans le même ordre d'idées, M. LoIsonLERUSTE et al. (2016) concluent que la réussite de la GJ dépend de la capacité des conseillers à fournir une assistance soutenue et adaptée à la population visée. C'est un élément fondamental, dans la mesure où cette population se caractérise souvent par des handicaps sociaux, scolaires, économiques, physiques et psychosomatiques.

Une cinquième condition indispensable à la mise en place efficace des programmes de GJ est d'assurer des ressources suffisantes. C'est vrai en ce qui concerne les moyens des SPE (coûts administratifs) et c'est aussi le cas pour les coûts opérationnels des programmes. Par exemple, il s'avère capital d'assurer que le budget alloué à ces politiques puisse bénéficier d'assez de flexibilité pour que les programmes soient en mesure de s'adapter aux cycles économiques (ESCUDERO et al., 2017). Le cas des SPE finnois a fourni aux observateurs un bon exemple de l'importance de cette flexibilité, quand la hausse rapide du nombre des jeunes chômeurs au cours de la récente crise économique a mis à l'épreuve sa capacité à produire une réponse efficace (ILO, 2012). Enfin, établir des projections précises des coûts administratifs et opérationnels liés à la mise en œuvre de la GJ est une étape importante, mais complexe, dans la mesure où ce calcul implique des investissements matériels, humains et organisationnels. Dans le cas français, par exemple, l'analyse de la mise en place de la GJ dans plusieurs délégations a montré que 
le budget alloué ne représentait que $80 \%$ du coût réel, souvent en raison de difficultés à anticiper les coûts réels qu'implique une mise en place efficace (FARVAQUE et al., 2016).

Sixième et dernier point : il est essentiel de s'assurer que les bénéficiaires respectent leurs obligations. Certaines études empiriques montrent en effet que l'augmentation des taux d'accès à l'emploi chez les participants résulte en partie de «l'effet de menace » associé aux mesures d'activation (GRAVERSEN, vAN OURS, 2008). Cet effet varie selon les caractéristiques de la GJ : il peut consister en une vraie réduction des prestations si les jeunes refusent des offres d'emploi ou de formation ; être associé au fait que le chômage soit plus coûteux - dans la mesure où les jeunes doivent consacrer du temps à participer à ces mesures d'activation - ou encore, pour les jeunes qui décident de ne pas participer, être lié à la stigmatisation que peut représenter le fait de ne pas prendre part à un programme généreux. Cet effet de menace est renforcé par le choix d'une approche basée sur les droits pour la conception de la GJ : ce principe implique dans la pratique que la participation à ces programmes soit « semi-obligatoire ».

\section{Évaluation de la mise en ouvre de la GJ : réussites et difficultés}

Les États membres éligibles à bénéficier de l'IEJ ont été incités par le Conseil européen à présenter leurs plans de mise en œuvre de la GJ avant la fin de l'année 2013, dans l'objectif de les amorcer en 2014 (EuROPEAn CounCIL, 2013). Malgré quelques retards, tous les pays qui souhaitaient bénéficier de l'IEJ ont présenté ces plans entre décembre 2013 et mars 2014. Les autres États membres (qui ne bénéficiaient pas de l'IEJ) ont été encouragés à adopter des plans similaires en 2014. Ainsi, l'Autriche, le Danemark, l'Estonie, la Finlande, l'Allemagne, le Luxembourg, Malte et les Pays-Bas ont lancé leurs projets au cours du premier semestre $2014^{15}$. Au cours des années 2014 et 2015 , près de la moitié des pays ( 13 sur les 28 de l'UE) ont même soumis des versions révisées des plans qu'ils avaient présentés.

Trois ans plus tard, les pays européens ont avancé dans la mise en œuvre de leurs plans nationaux de GJ. Les données disponibles (EC, 2016b) nous permettent d'évaluer les résultats de cette mise en œuvre, jusqu'en octobre 2016 (date des informations les plus récentes dont nous disposons). Nous comparons aussi ces données avec celles des mesures prévues par les pays, dans leurs plans de mise en œuvre ${ }^{16}$; nous regardons en particulier si les pays ont tenu compte des facteurs de réussite présentés plus haut tout en identifiant les points positifs et les difficultés qui subsistent encore.

15. Certains de ces pays, dont l'Autriche et le Danemark, disposaient déjà de garanties jeunesse, qu'ils ont remodelées et adaptées pour qu'elles répondent à la recommandation de la CE. Les plans de mise en œuvre présentés contenaient dès lors les traits principaux d'une garantie jeunesse conforme aux indications européennes.

16. Les informations prises en compte dans cette analyse, concernant les plans de mise en œuvre de la GJ, se limitent aux plans disponibles en ligne. 
Verónica ESCUDERO et ses co-auteur·e·s (2017) ont fourni une première évaluation des progrès accomplis dans la mise en œuvre des plans de GJ au niveau national, particulièrement sur les questions liées au type de politique adoptée. L'objectif de cette partie est d'actualiser et de compléter ces analyses, ainsi que d'offrir de nouvelles perspectives concernant la prise en compte des facteurs de réussite. Nous examinerons tout d'abord si les pays de l'UE ont opté pour des critères d'éligibilité clairs dans leurs plans de mise en œuvre de la GJ, puis nous verrons si ces pays ont fixé un délai maximal pour subvenir aux besoins de la jeunesse et, surtout si, dans la pratique, ils ont rempli l'objectif d'exécuter leur intervention dans un délai convenable. Nous regarderons ensuite en détail la série d'initiatives prévue et mise en œuvre dans ces pays, pour voir s'ils ont été en mesure de fournir une gamme complète de mesures d'activation. La fin de cette partie s'intéressera aux trois autres prérequis nécessaires à la réussite des programmes de GJ, à savoir : des cadres institutionnels appropriés, des ressources suffisantes et la disponibilité de mécanismes qui puissent assurer que les bénéficiaires respectent leurs obligations.

\section{Publics cibles et éligibilité dans le cadre des plans de GJ}

Par la GJ, les pays s'engagent à aider les jeunes de moins de 25 ans dans les quatre mois suivant leur entrée au chômage ou la fin de leurs études. Par rapport à cet engagement, 11 pays ont suivi la recommandation de la CE (tableau 1) en ciblant les moins de 25 ans, 15 pays ont étendu l'éligibilité et mis en œuvre une GJ ciblant les jeunes de moins de 30 ans et la France comme les Pays-Bas ont établi une limite d'âge intermédiaire $^{17}$ (respectivement à 26 et 27 ans).

De plus, au regard des critères d'éligibilité à la GJ, la plupart des pays ont ciblé les jeunes Neets et exigent qu'ils soient inscrits auprès d'un prestataire de la GJ (typiquement un SPE national ou régional). La seule exception est la Slovénie, qui demande que les jeunes soient sans emploi. Les caractéristiques des groupes ciblés ne s'étendent souvent pas au-delà de ces critères généraux. On compte cependant sept pays qui ont défini des sous-groupes spécifiques à l'intérieur de la population généralement ciblée, notamment les femmes, les personnes présentant des niveaux d'instruction particuliers ou des handicaps, ou encore les populations Roms (EC, 2016c).

En résumé, tous les pays de l'Union européenne ont établi des critères d'éligibilité clairs et, point très important, ont souvent ciblé les jeunes les plus vulnérables sur le marché du travail.

17. Informations recueillies à partir des plans de mise en œuvre et des fiches par pays disponibles en ligne (CE 2016b et 2016c ; EsCudero, LóPEZ Mourelo, 2015). 
TABLEAU 1 - Caractéristiques principales des plans nationaux de Garantie jeunesse, par pays

\begin{tabular}{|c|c|c|c|c|c|}
\hline Pays & $\begin{array}{l}\text { Période } \\
\text { maximale }\end{array}$ & $\begin{array}{l}\text { Groupe } \\
\text { d'âge }\end{array}$ & $\begin{array}{l}\text { Groupe } \\
\text { principalement } \\
\text { ciblé : Neet }\end{array}$ & $\begin{array}{l}\text { Chômeurs } \\
\text { de longue } \\
\text { durée ciblés } \\
\text { spécifiquement }\end{array}$ & Cibles spécifiques \\
\hline $\begin{array}{l}\text { Allemagne, } \\
\text { Autriche, } \\
\text { Belgique, } \\
\text { Chypre, } \\
\text { Irlande, } \\
\text { Luxembourg, } \\
\text { Malte, } \\
\text { Suède }\end{array}$ & $\begin{array}{l}-4 \text { mois } \\
\text { - Autriche, } \\
\text { Allemagne, } \\
\text { Suisse: } \\
3 \text { mois }\end{array}$ & $<25$ ans & $\checkmark$ & $\times$ & \\
\hline Hongrie & 4 mois & $<25$ ans & $\checkmark$ & $\checkmark$ & \\
\hline Finlande & 3 mois & $<25$ ans & $\checkmark$ & $\times$ & $\begin{array}{l}\text { Niveaux particuliers d'instruction } \\
\text { (diplômés récents âgés de moins } \\
\text { de } 30 \text { ans) }\end{array}$ \\
\hline Roumanie & 4 mois & $<25$ ans & $\checkmark$ & $x$ & $\begin{array}{l}\text { - Populations roms } \\
\text { - Femmes }\end{array}$ \\
\hline France & 4 mois & $<26$ ans & $\checkmark$ & $x$ & \\
\hline Pays-Bas & 4 mois & $<27$ ans & $\checkmark$ & $\times$ & \\
\hline $\begin{array}{l}\text { Croatie, } \\
\text { République } \\
\text { tchèque, } \\
\text { Danemark, } \\
\text { Estonie, } \\
\text { Grèce, } \\
\text { Lituanie, } \\
\text { Portugal }\end{array}$ & $\begin{array}{l}-4 \text { mois } \\
\text { - Danemark : } \\
3 \text { mois }\end{array}$ & $<30$ ans & $\checkmark$ & $\times$ & \\
\hline $\begin{array}{l}\text { Lettonie, } \\
\text { Slovaquie }\end{array}$ & 4 mois & $<30$ ans & $\checkmark$ & $\checkmark$ & \\
\hline Slovénie & 4 mois & & Chômeurs & $\checkmark$ & \\
\hline Bulgarie & 4 mois & $<30$ ans & $\checkmark$ & $\checkmark$ & $\begin{array}{l}\text { - Populations roms } \\
\text { - Niveaux particuliers d'instruction }\end{array}$ \\
\hline Italie & 4 mois & $<30$ ans & $\checkmark$ & $\checkmark$ & Populations roms \\
\hline Pologne & 4 mois & & $\checkmark$ & $\checkmark$ & Individus présentant un handicap \\
\hline Espagne & 4 mois & $<30$ ans & $\checkmark$ & $x$ & $\begin{array}{l}\text { - Femmes } \\
\text { - Individus présentant un handicap } \\
\text { - Niveaux particuliers d'instruction }\end{array}$ \\
\hline Royaume-Uni & 4 mois & $<30$ ans & $\checkmark$ & $\checkmark$ & $\begin{array}{l}\text { - Individus présentant un handicap } \\
\text { - Niveaux particuliers d'instruction }\end{array}$ \\
\hline
\end{tabular}

Source : Informations compilées par les auteures, à partir des plans de mise en œuvre de la GJ et des fiches par pays disponibles en ligne : http://ec.europa.eu/social/main.jsp?catId=1161\&langId=en ; UE (2016b et 2016c). 


\section{L'objectif de l'intervention précoce}

Tous les pays ont suivi la recommandation européenne sur le délai maximal de quatre mois pour que les jeunes reçoivent une proposition d'emploi ou une offre de formation. Certains pays scandinaves (Danemark, Finlande et Suède), ainsi que l'Autriche et l'Allemagne, ont même opté pour un délai plus court de trois mois.

Les données de suivi disponibles concernant la GJ suggèrent que la plupart des pays rencontrent des difficultés pour respecter cet objectif. Le graphique 1 indique en particulier que $57 \%$ des jeunes européens inscrits dans un programme national de GJ en 2015 étaient enregistrés depuis plus de quatre mois. Cette situation ne diffère pas de manière significative entre les femmes et les hommes (respectivement $58,3 \%$ et $55,9 \%$ ) ou selon les groupes d'âge (58,6\% chez les $15-19$ ans contre $56,4 \%$ chez les 20-24 ans), mais il existe de grandes différences selon les États membres (graphique 2). À titre d'exemple, avec moins de $30 \%$ des participants à la GJ entrés au-delà du délai de quatre mois, Malte, l'Estonie et la Hongrie sont les plus performants en termes d'intervention précoce. À l'opposé du spectre, des pays comme l'Irlande et la Slovaquie comptent plus de $60 \%$ de participants à la GJ enregistrés après plus de quatre mois. Cette situation est même plus frappante dans le cas de la France, avec plus de $75 \%$ des participants à la GJ enregistrés au-delà de la période maximale. Cette difficulté à effectuer des interventions dans les quatre mois peut indiquer la présence

GRAPHIQUE 1 - Proportion des participants à la GJ enregistrés pendant plus de 4 mois, par pays et par classe d'âge (2015)

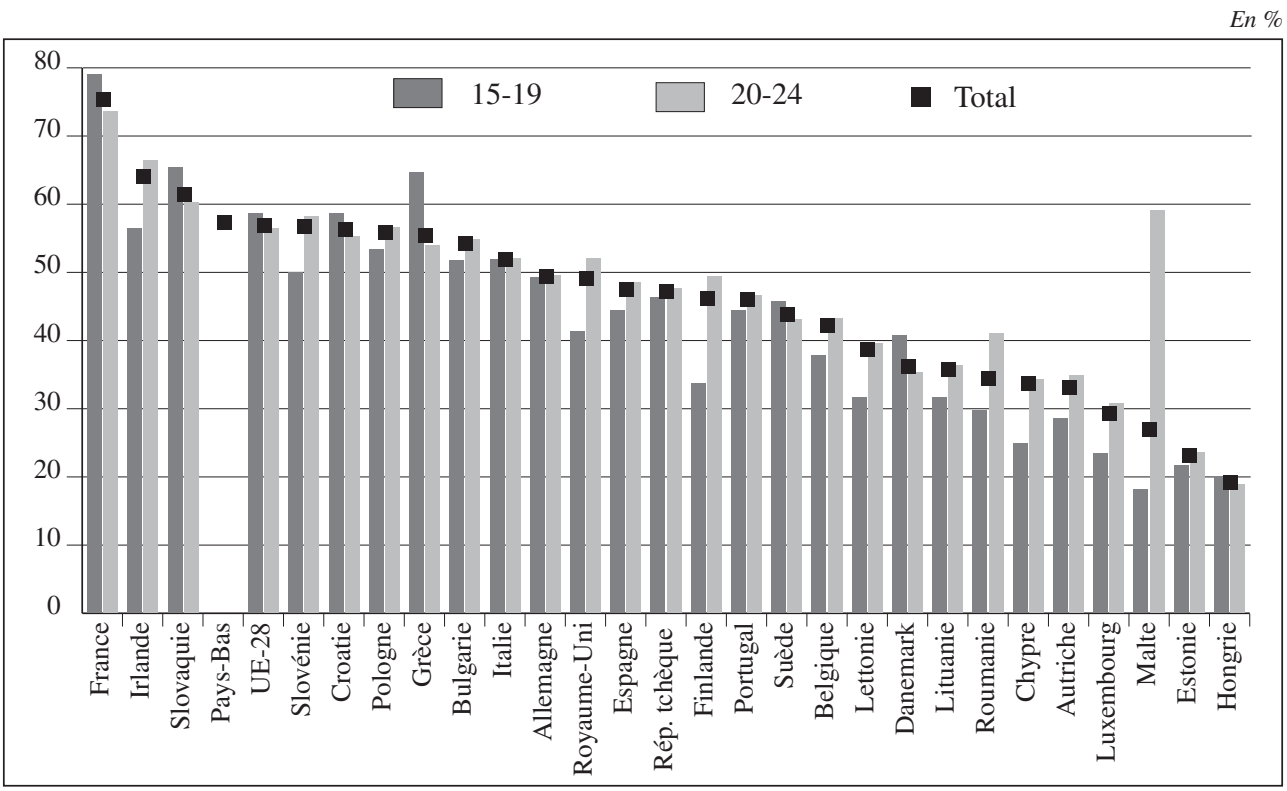

Note : données par groupes d'âges non disponibles pour les Pays-Bas.

Source : base de données dans le cadre d'indicateurs de la GJ. 
GRAPHIQUe 2 - Proportion des participants à la GJ enregistrés pendant plus de 4 mois, par pays et par sexe (2015)

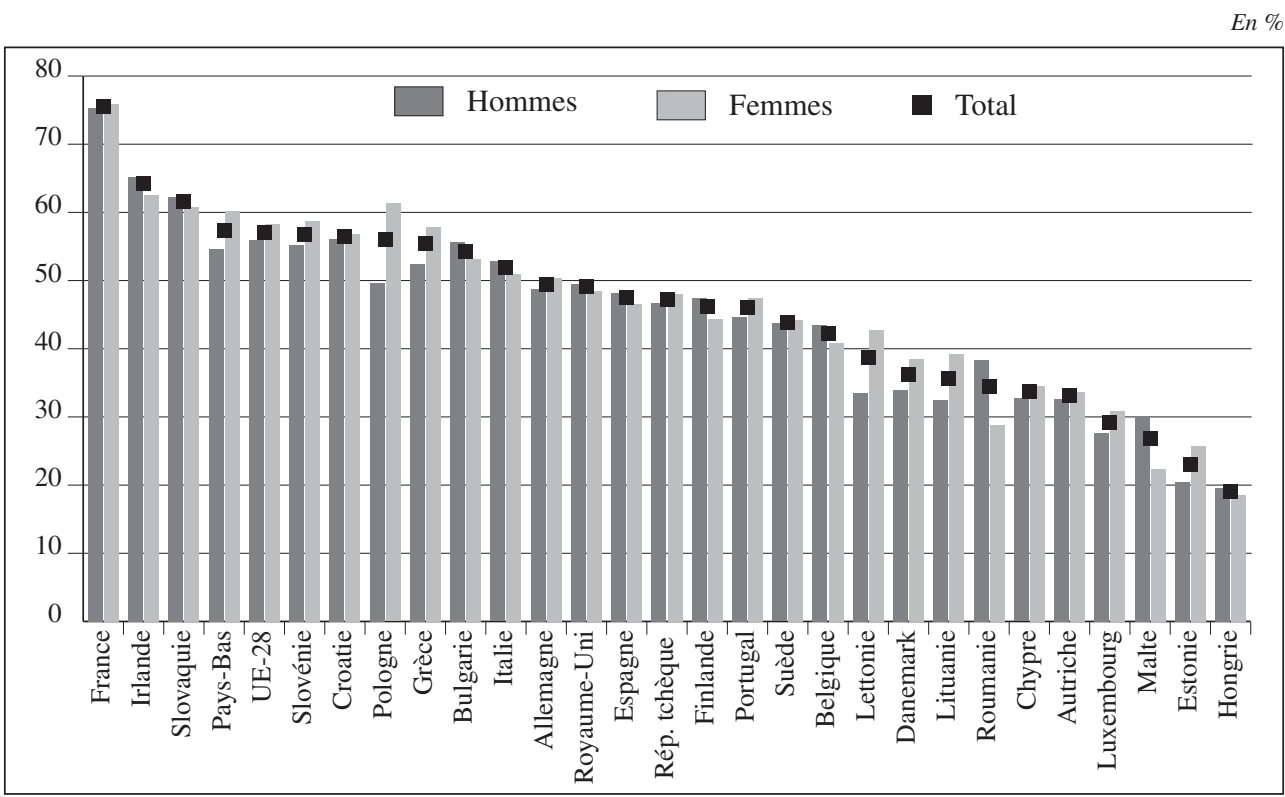

Source : base de données dans le cadre d'indicateurs de la GJ.

d'une catégorie assez importante de jeunes difficiles à placer, mais aussi le manque de cadres institutionnels adéquats pour les aider efficacement.

\section{Les mesures et initiatives mises en œuvre dans le cadre de la GJ}

Des mesures et initiatives très diverses ont été incluses dans le cadre de la GJ. Elles peuvent être réparties en quatre catégories : 1) programmes de formation scolaire et professionnelle ; 2) mesures de remédiation en cas de décrochage scolaire ; 3) services d'intermédiation vis-à-vis du marché du travail ; 4) PAMT visant à agir sur la demande de travail, comme la création directe d'emplois, les subventions à l'embauche et les incitations à la création d'entreprises.

Dans le but d'améliorer la qualification des jeunes, tous les États membres intègrent des programmes de formation scolaire et professionnelle dans leurs plans de mise en œuvre (graphique 3). Les outils les plus répandus sont les cursus d'enseignement et de formation professionnels, les stages et les contrats d'apprentissage. Comme le manifeste l'accent mis sur l'exécution de ces politiques, les pays accordent une forte priorité aux mesures d'amélioration des qualifications. De fait, tous les pays, sauf la Hongrie et l'Italie, ont pris des initiatives en la matière. Plus spécifiquement, la priorité est donnée à l'offre de stages et de contrats d'apprentissage, mesures actuellement prises dans $85 \%$ des pays. 
GRAPHIQUE 3 - Nombre de pays ayant planifié et mis en œuvre chaque type de mesures

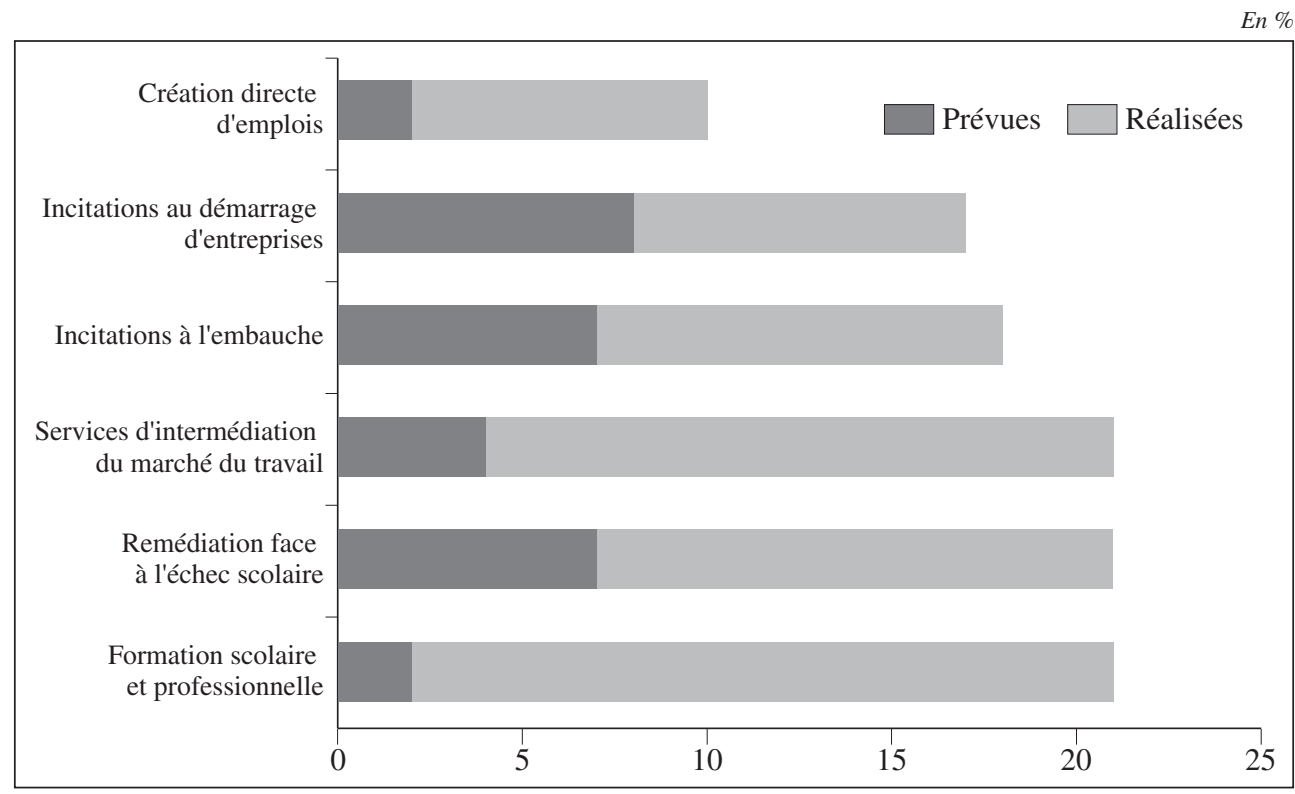

Note : les informations sur la Bulgarie, Chypre, la Grèce, la Slovaquie, la Slovénie et le Royaume-Uni, non disponibles en ligne, ne sont pas reproduites.

Source : informations compilées par les auteures à partir de EC (2016b), EscudERo et al. (2016) et des plans additionnels de mise en œuvre disponibles en ligne.

Les mesures visant à réduire le décrochage scolaire et à améliorer l'achèvement des études sont également très répandues dans les plans nationaux. Cela est cohérent étant donné le pourcentage élevé de jeunes chômeurs appartenant à des groupes vulnérables, qui manquent de compétences techniques, voire élémentaires. En comblant ces lacunes en compétences de base, on espère réduire leur degré de vulnérabilité et surtout leur permettre de tirer profit d'autres programmes d'activation visant à augmenter leur employabilité. Ce type d'intervention inclut principalement des initiatives pour contrer l'abandon précoce de la scolarité, tandis que les mesures de reprise de scolarité, à l'intention de ceux qui ont déjà abandonné l'école, sont moins présentes. Malgré l'intérêt de ces interventions, seuls neuf des vingt-et-un pays étudiés ${ }^{18}$ se sont à ce jour efforcés de les instaurer, ce qui s'explique par des facteurs sociaux et économiques, comme le fait que ces mesures soient le plus souvent conçues pour des jeunes particulièrement difficiles à atteindre et à aider, ou encore par le fait que la mise en œuvre de ces politiques requiert souvent une réforme du système éducatif.

Tous les États membres ont prévu de renforcer les services d'intermédiation sur le marché du travail, pour augmenter l'efficacité de la recherche d'emploi des jeunes et faciliter l'adéquation entre l'offre et la demande de main-d'œuvre. Le rôle central que

18. Ce sont les vingt-et-un pays pour lesquels les plans de mise en œuvre de la GJ sont disponibles en ligne. 
joue cette intermédiation pour une mise en œuvre réussie des programmes nationaux de GJ est illustré par le fait que les pays ont entrepris la mise en application des mesures prévues - à la seule exception de la République tchèque, du Danemark, des Pays-Bas et de la Suède. En observant plus attentivement la mise en œuvre effective de la GJ, on observe toutefois que les pays membres se sont surtout attachés à renforcer leur SPE, plutôt que de fournir des services additionnels d'intermédiation. Douze des dix-sept pays ayant mis en œuvre des services d'intermédiation pour les jeunes en GJ avaient par ailleurs déjà entrepris une réforme de leur SPE. La Croatie, la France et le Luxembourg fournissent de bons exemples de réformes significatives dans ce domaine. Plus spécifiquement, ces trois pays ont revu les activités de leur SPE, lancé de nouvelles procédures et augmenté le personnel du SPE, en allouant des ressources exclusivement destinées à la mise en œuvre de la GJ. Les efforts pour améliorer les capacités du SPE au niveau national ont été soutenus par les activités d'apprentissage mutuel organisées au niveau transnational par le Réseau européen des services publics de l'emploi.

L'importance accordée par les pays dans la mise en œuvre des PAMT visant à augmenter la demande de travail varie davantage. Les incitations à l'embauche sont l'un des types les plus communs de PAMT incluses dans les plans de GJ, intégrées par pratiquement tous les pays, à l'exception de l'Autriche, de la République tchèque et de la France. Ces incitations prennent généralement la forme de subventions à l'emploi ou de réductions du coût de l'embauche, par l'intermédiaire de réductions de cotisations sociales. Il semble plus simple de recourir à ce type de mesures qu'à d'autres dispositifs ciblés sur la demande de travail, tels que les programmes de soutien aux jeunes entrepreneurs. Il n'est donc pas surprenant que $60 \%$ des pays aient amorcé la mise en place d'incitations à l'embauche, en particulier pour augmenter les perspectives d'emploi des jeunes peu qualifiés, comme c'est le cas des mesures instaurées en Belgique, en Estonie et au Luxembourg.

Les incitations à la création d'entreprises et les programmes de création directe d'emplois sont en revanche bien moins répandus dans les plans de mise en œuvre de la GJ. Les programmes en faveur de l'entrepreneuriat des jeunes proposent généralement un soutien financier à la création de nouvelles entreprises, souvent complété par la formation et un suivi, soutien nécessaire pour accroître le taux de survie des entreprises. Les programmes de création directe d'emplois mis en œuvre dans le cadre de la GJ consistent souvent à créer des postes de courte durée dans le secteur public, pour donner aux jeunes l'opportunité de mener à bien des projets pour les communautés locales. Il existe d'importantes différences selon les pays quant à la préférence apportée à l'un ou l'autre type d'intervention : si la plupart des pays européens ont prévu d'inclure des programmes d'encouragement à l'entrepreneuriat des jeunes, seuls dix pays envisagent de mettre en œuvre des programmes d'emplois publics temporaires. 


\section{Un cadre institutionnel adapté, des ressources suffisantes et des dispositions pour assurer le respect des règles}

Ayant analysé les trois premiers facteurs de réussite, nous proposons maintenant de déterminer si les autres éléments clés de réussite sont présents dans les différents pays, à savoir : le développement de cadres institutionnels adaptés, une allocation suffisante de ressources et l'instauration de règles visant à assurer que les bénéficiaires respectent leurs obligations.

Les analyses montrent en premier lieu que la plupart des pays ont concentré leurs efforts sur la création de cadres institutionnels adaptés, mobilisant une vaste gamme d'acteurs dans la mise en œuvre la GJ. Dans la grande majorité des cas, l'organe responsable de l'administration publique est le ministère du Travail, bien que les ministères de l'Éducation, des Affaires sociales ou de la Science et de la Recherche soient également impliqués. De plus, afin d'assurer les conditions nécessaires à la mise en place d'une GJ efficace, la plupart des pays ont prévu une modernisation de leur SPE : la grande majorité des pays a amélioré le ciblage et la gamme des services fournis par leur SPE ; deux tiers d'entre eux ont établi des cibles spécifiques pour mieux personnaliser certains services ; la moitié d'entre eux a augmenté la dotation en personnel de leur SPE. Enfin, le dialogue social a été un élément important dans l'établissement des programmes nationaux de GJ. Les partenaires sociaux semblent avoir été impliqués dans tous les pays, à un moment ou à un autre de la conception (dans plus de $91 \%$ des pays), de la mise en œuvre (dans plus de $78 \%$ des pays) ou de l'évaluation (dans plus de $65 \%$ des cas) des plans de GJ (EC, 2016a).

Les informations existantes permettent par ailleurs de mener une évaluation préliminaire des efforts consentis dans la plupart des pays pour fournir aux plans nationaux de GJ des ressources financières suffisantes. Nous estimons pour cela les sommes que les pays membres ont réellement dépensées, par bénéficiaire, pour les initiatives de GJ pendant les trois premières années de mise en œuvre (graphique 4). Nous comparons ces valeurs avec, d'une part, les dépenses prévues indiquées par les pays dans leurs plans de mise en œuvre et, d'autre part, les dépenses recommandées à partir du modèle suédois ${ }^{19}$, selon les modes de calcul empruntés à Verónica EsCUDERO et Elva LóPEZ Mourelo (2015) et à V. EsCudERo et ses co-auteur.e-s (2017). Le graphique 4 présente les résultats de cette analyse, qui met en lumière trois résultats. Tout d'abord, il existe une variation significative selon les pays dans les dépenses prévues par participant éligible, qui vont de 13300 euros (PPA) par participant en Hongrie à seulement 115 euros (PPA) par participant en Croatie. Deuxièmement, plus de $60 \%$ des pays ont prévu des dépenses inférieures au seuil recommandé. Cette divergence est particulièrement importante dans le cas de la Croatie et de la Belgique, qui présentent respectivement une dépense douze fois et sept fois inférieure à celle

19. Nous avons estimé que le coût total qui devait être recommandé pour le plan de GJ de l'UE à 28 était d'environ 45,4 milliards d'euros en 2014 (EsCudERo, LóPEZ Mourelo, 2015). Ces dépenses recommandées se fondent sur le coût du programme suédois, qui, en 2010, s'élevait à 6000 euros par participant, auquel s'ajoutent les coûts administratifs (environ 600 euros par participant). 


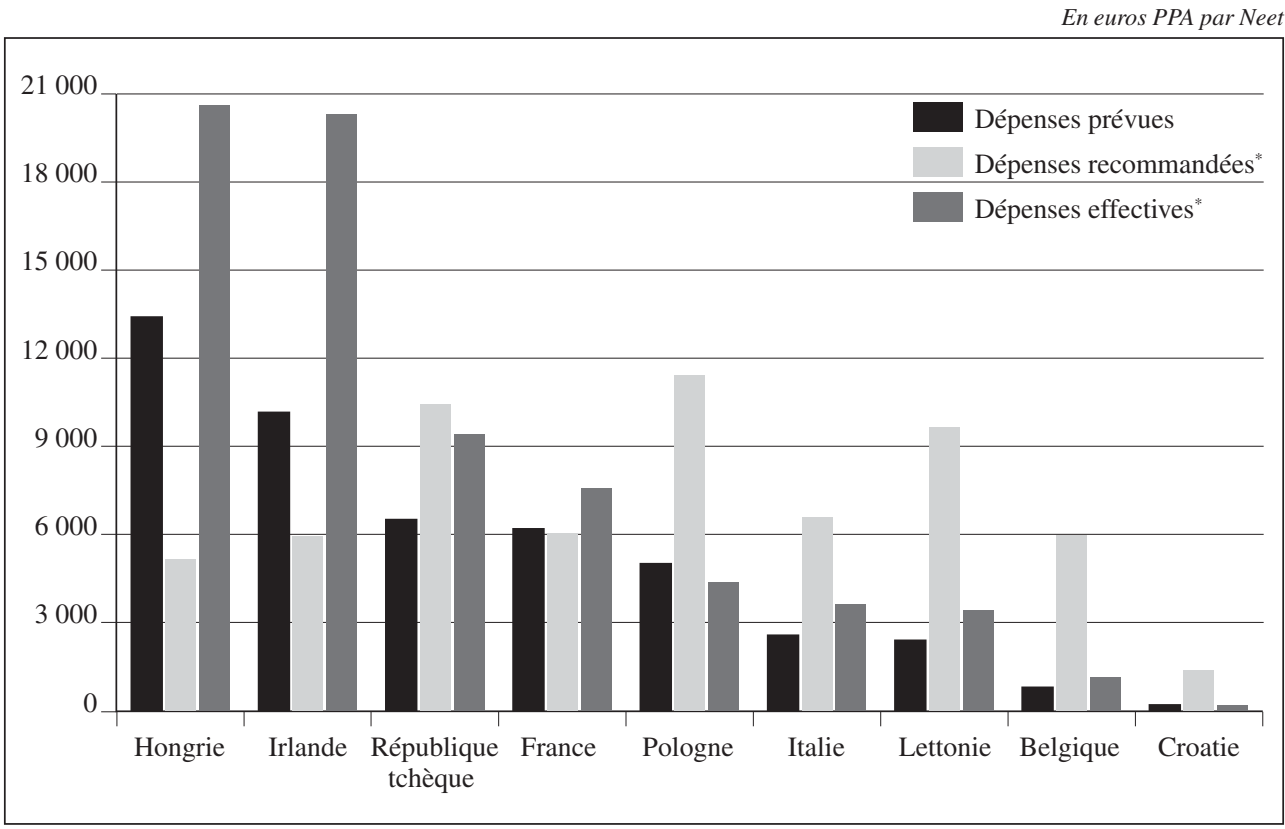

Note : * estimations tirées d'une comparaison entre les allocations prévues de l'IEJ que les pays membres ont reportées dans leurs plans de mise en œuvre et les allocations financières approuvées pour les opérations de l'IEJ (EC, 2016b). Le ratio entre ces deux valeurs a ensuite été appliqué aux dépenses globales prévues de l'IEJ pour estimer les sommes réelles. Toutes les dépenses sont ensuite divisées par le nombre de bénéficiaires éligibles, afin de s'assurer que les différences ne reflètent que des changements de dépenses. Source : calculs effectués par les auteures à partir d'Eurostat et des plans de mise en œuvre de la GJ disponibles en ligne (http://ec.europa. eu/social/main.jsp?catId=1161\&langId=en\&intPageId=3347).

qui est recommandée. Réciproquement, certains pays, dont la Hongrie et l'Irlande, ont soumis dans leurs plans de mise en œuvre des budgets significativement plus élevés que les montants recommandés. Troisièmement, tous les pays pour lesquels l'information est disponible ont en fait alloué davantage de fonds aux interventions de GJ qu'initialement prévu, à l'exception de la Pologne. Malheureusement, malgré ces améliorations, les pays qui avaient prévu des dépenses inférieures aux niveaux recommandés n'ont pas réussi à faire de progrès substantiels pour réduire cet écart. La seule exception est la République tchèque, où le montant dépensé par bénéficiaire pendant les trois premières années de mise en œuvre était seulement inférieur de 9,5\% à la somme recommandée.

La GJ a un coût mais l'inaction a également un prix, qui s'avère en réalité plus élevé que les dépenses recommandées. Une recherche récente estime que les allocations, ainsi que les revenus et impôts perdus des jeunes Neet équivaudraient à 162 milliards d'euros, ou 1,2 \% de PIB par an (MASCHERINI et al., 2014).

Enfin, dans le cadre des dispositions destinées à assurer le respect des engagements de la part des bénéficiaires de la GJ, la plupart des pays appliquent cette obligation 
mutuelle pour les chômeurs enregistrés, bien que cela ne relève pas spécifiquement de leurs plans nationaux de GJ (EC, 2016b). Selon les informations relatives à tous les pays européens, la perte d'allocations parmi les participants de la GJ en 2015 s'explique par le fait que ces derniers refusent des propositions a priori conformes à leur profil ou qu'ils ne respectent pas des obligations, comme celle de se rendre aux entretiens (EC, 2016b). Cependant, dans certains pays, les allocations sont limitées dans le temps. C'est le cas en France, où les droits aux services de GJ expirent au terme d'une période de dix-huit mois. De façon intéressante, comme le montrent M. LoIsONLERUSTE et ses co-auteur.e.s (2016) pour ce pays, les bénéficiaires ont tendance à bien accepter les obligations liées à la participation au programme, car ils reconnaissent les bénéfices qu'elle leur apporte.

\section{Les difficultés dans la mise en œuvre de la GJ européenne au niveau national}

La GJ européenne a joué un rôle moteur dans les changements au niveau politique et institutionnel. De fait, entre 2013 et 2015, les États membres ont au total adopté 132 réformes du marché du travail visant les jeunes (EC, 2016b). Les expériences sont très différentes selon les pays. Plusieurs d'entre eux ont dû bâtir de toutes pièces les bases d'une mise en œuvre de la GJ, tandis que d'autres disposaient déjà de politiques et d'institutions centrées sur les difficultés des jeunes vis-à-vis du marché du travail, qui devaient simplement être adaptées pour remplir les engagements spécifiques de la GJ (comme l'intervention précoce ou l'attention particulière portée aux Neet).

Selon une analyse de la Communauté européenne (EC, 2016a), on peut définir trois catégories de pays quant au nombre de réformes entreprises pour l'emploi des jeunes, en lien avec le contexte institutionnel et les conditions macroéconomiques qu'ils connaissaient au moment de l'adoption de la recommandation sur la GJ (graphique 5). Le premier groupe (groupe A) comporte des pays qui avaient déjà mis en place des politiques bien établies, largement en adéquation avec les recommandations sur la GJ, au cours des quinze années précédentes. Le deuxième groupe (groupe $\mathrm{B}$ ) comporte des pays qui disposaient déjà de politiques complètes pour aider les jeunes, mais où les jeunes rencontraient des difficultés majeures lorsque la décision de mettre en œuvre la GJ avait été adoptée ; ces pays reçoivent une aide financière substantielle et la GJ a donné un nouvel essor aux politiques envers les jeunes. Le troisième groupe (groupe C) rassemble les pays où les réformes ont été plus limitées, malgré les difficultés significatives rencontrées par les jeunes sur le marché du travail.

Les difficultés à mettre en œuvre la GJ ont été particulièrement prononcées dans certains pays comme la République tchèque, la Roumanie et la Slovaquie, pays qui avaient prévu une large gamme de mesures pour améliorer la situation des jeunes sur le marché du travail, mais où la plupart des initiatives sont restées au stade de 
GRAPHIQUE 5 - Nombre de réformes concernant les jeunes, par groupe de pays**

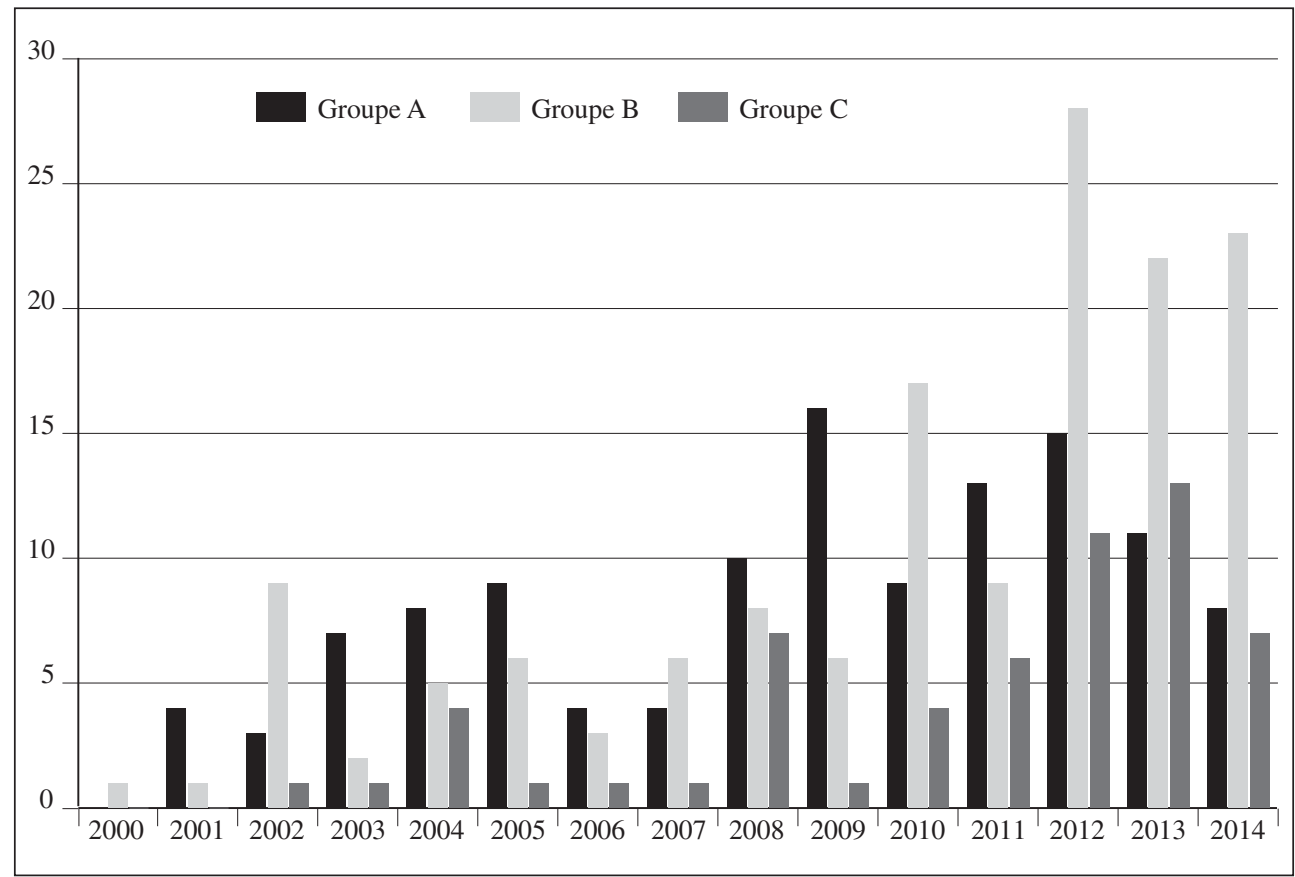

Note : * les pays ont été regroupés selon leur capacité (de la plus forte pour le groupe $\mathrm{A}$ à la moins forte pour le groupe C) à mettre en œuvre des réformes qui les rapprochent des recommandations relatives à la GJ.

Groupe A : Autriche, Danemark, Estonie, Finlande, Allemagne, Irlande, Malte, Pays-Bas, Suède, Royaume-Uni.

Groupe B : Belgique, Bulgarie, Croatie, France, Italie, Lettonie, Lituanie, Pologne, Portugal, Slovénie.

Groupe C : Chypre, République tchèque, Grèce, Roumanie, Slovaquie, Espagne.

développement. Par conséquent, la proportion de mesures effectivement exécutées dans ces pays est significativement plus basse que la moyenne. Pour expliquer ce développement contrarié, on peut mettre l'accent sur la capacité limitée des SPE à identifier les Neets non enregistrés et à fournir des services individualisés de qualité. Il semblerait en outre nécessaire de renforcer la coopération entre le SPE, les écoles et les autres centres de formation, ainsi qu'avec les partenaires sociaux et les autres parties prenantes (EC, 2016b).

Selon les configurations institutionnelles nationales et le contexte économique, des différences se sont ainsi fait sentir dans l'effort consenti par les pays membres afin de remplir les exigences établies par la GJ. Par exemple, en France, les services d'intermédiation en matière d'emploi, en particulier les programmes de conseils en termes de carrière, jouent un rôle croissant depuis 2000. Certaines mesures innovantes - comme le programme TRACE (Trajet d'accès à l'emploi), qui proposait un suivi individuel de dix-huit mois aux jeunes chômeurs en risque d'exclusion sociale - ont été une source majeure de connaissances et d'expertise pour la mise en œuvre efficace des initiatives incluses dans la GJ (DAREs, 2016). Cependant, il peut s'avérer difficile 
d'adapter les mesures et les institutions déjà en place aux exigences de la GJ (voir, par exemple, FARVAQUE et al., 2016). En particulier, la mise en œuvre du programme national français de GJ a pris appui sur un nouveau cadre de référence promu par le ministère du Travail, au sein duquel le contact avec les employeurs potentiels devait, dans la mesure du possible, devenir pérenne et intervenir en amont. Le fait de travailler en étroite collaboration avec les jeunes et les entreprises a aussi changé en profondeur le sens, l' approche et l'organisation du travail des personnes chargées de conseiller les jeunes, et a nécessité des ressources financières additionnelles, à la fois pour accroître le nombre de conseillers et pour leur fournir les compétences nécessaires pour agir efficacement dans ce nouveau cadre.

Enfin, il est important de noter que même dans le cas où les pays membres avaient déjà établi des mesures pertinentes concernant l'emploi des jeunes avant l'adoption de la GJ, il a fallu, pour mettre en œuvre les plans nationaux de ce dispositif, modifier la conception et la conduite de ces politiques pour y intégrer les Neets qui n'étaient ni inscrits ni identifiés. Une des difficultés rencontrées par les pays membres, lorsqu'ils s'efforcent de suivre les instructions de la recommandation de la Communauté

\section{GRAPHiQue 6 - Taux de Neets et proportion de Neets touchés par la GJ}

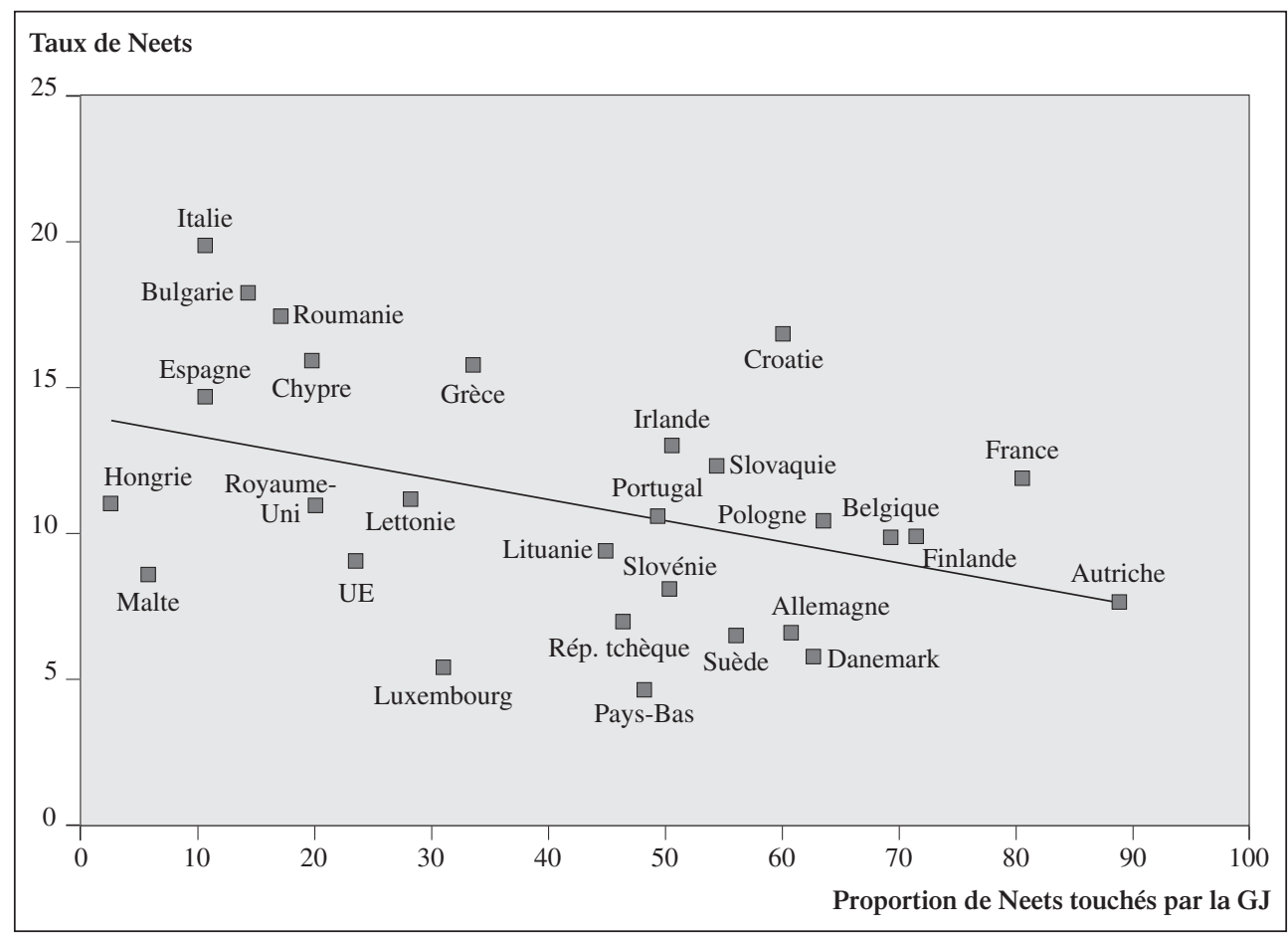

Source : calculs effectués par les auteures, à partir d'Eurostat et des fiches d'informations par pays dans « La Garantie pour la jeunesse et l'Initiative pour l'emploi des jeunes - bilan des trois premières années » ("Youth Guarantee and Youth Employment Initiative - three years on"). 
européenne, est de parvenir à toucher cette catégorie de jeunes, souvent isolés du fait de leur faible niveau d'instruction, leur situation familiale et leur contexte personnel difficile. En 2016, la proportion de Neets aidés par la GJ était très variable (graphique 6) : plus de $80 \%$ en Autriche (88,7\%) et en France (80,5\%), mais moins de $10 \%$ en Hongrie $(2,6 \%)$ et à Malte $(5,6 \%)$. Le fait le plus préoccupant est que la proportion de Neets qui bénéficie de la GJ est généralement plus basse dans les pays où le taux de Neets est le plus élevé. Pour assurer une mise en œuvre complète de la GJ, il est donc crucial de développer des mécanismes adaptés pour atteindre, identifier et assister les groupes de Neets les plus vulnérables, qui sont aussi les plus difficiles à toucher. C'est d'autant plus important que la proportion de Neets a connu peu de changements ces trois dernières années, en particulier dans les pays (comme l'Italie ou la Roumanie) où cette part était élevée en 2013 (annexe 2).

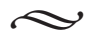

Le lancement de la GJ européenne en avril 2013 a suscité un grand intérêt en Europe, non seulement en raison du contexte - elle a été lancée à un moment où se faisait fortement sentir la nécessité d'une réponse urgente et coordonnée face au niveau du taux de chômage des jeunes - mais aussi en raison de ses traits innovants. Dans le cadre de la GJ, tous les États membres de l'UE s'engageaient à assurer à tous les jeunes chômeurs une poursuite d'études, un apprentissage, une formation ou une offre d'emploi dans un délai maximal de quatre mois. Afin de mettre en pratique un tel engagement, il a fallu consacrer d'importants efforts pour déployer des politiques, adapter les cadres institutionnels existants et allouer des ressources suffisantes. En prenant appui sur les données récentes relatives à la mise en œuvre des plans nationaux de GJ - plans de mise en œuvre publiés par les pays européens, documents récents sur les progrès accomplis dans cette instauration - cet article a cherché à éclairer les éléments de réussite et les difficultés rencontrées par les pays membres pour atteindre ces objectifs ambitieux.

Les résultats empiriques issus de pays ayant une longue expérience dans la mise en œuvre de garanties jeunesse (comme les pays scandinaves) suggèrent que ces interventions sont une manière efficace de répondre au chômage des jeunes. Conçues et instaurées correctement, elles peuvent produire des effets significatifs à un coût modéré. Plus particulièrement, cet article a identifié des éléments essentiels au fonctionnement efficace de ces programmes : une intervention précoce, des cadres institutionnels adaptés, des ressources suffisantes et la mise à disposition de mécanismes incitatifs.

Trois ans après le lancement de la GJ, plusieurs résultats peuvent être mis en évidence. Tous les pays européens ont tout d'abord établi des critères d'éligibilité clairs et développé des mesures visant de manière spécifique les jeunes les plus vulnérables. Si l'on considère le vaste panel des mesures prévues dans le cadre de la GJ, leur mise en œuvre a par ailleurs été en général assez rapide (à l'exception de retards dans les incitations à l'égard du secteur privé). Enfin, les efforts accomplis pour créer des cadres institutionnels ou adapter les cadres existants, afin de satisfaire aux recommandations 
de la GJ, ont également été importants, en particulier pour moderniser les organismes nationaux de SPE et améliorer leur efficacité.

Mais l'observation des premières années de mise en œuvre de la GJ révèle aussi un certain nombre de difficultés qui s'imposent encore aux pays membres. Celles devant particulièrement attirer l'attention concernent l'insuffisance des ressources et l'incapacité à fournir systématiquement une intervention précoce. Ainsi, $60 \%$ des pays analysés ont prévu des dépenses en dessous des niveaux recommandés et, bien que certains aient en pratique alloué plus de ressources aux interventions liées à la GJ qu'initialement prévu, la majorité d'entre eux n'a pas réussi à faire des progrès substantiels pour combler cet écart. Cela signifie que ces pays devront prendre maintenant des engagements financiers plus importants, s'ils veulent tenir l'objectif visé de réduire le chômage des jeunes. La question de l'intervention précoce est également préoccupante. Ainsi, alors que tous les pays ont fixé à quatre mois le délai maximal pour aider les jeunes sans emploi, en pratique $57 \%$ des jeunes européens enregistrés dans un plan national de GJ en 2015 étaient sans emploi depuis plus de quatre mois. Dans le même ordre d'idées, le projet de GJ n'a pas été lancé aussi tôt qu'il aurait dû l'être (alors qu'un pic du taux de chômage dans l'UE a été atteint en 2010, la mise en œuvre de la GJ ne s'est produite que quatre à cinq ans plus tard). Cela crée une autre limite à l'efficacité de la GJ, la durée du chômage affaiblissant l'efficacité des politiques d'activation.

Enfin, les efforts engagés pour obéir aux recommandations de la GJ ont varié selon les pays. Les difficultés rencontrées diffèrent selon la façon dont ils ont ajusté leur démarche à la GJ, en créant de nouvelles politiques et institutions ou en adaptant des formes existantes, mais il existe peu d'informations à ce sujet. Cela constitue, de notre point de vue, un futur axe de recherches, qui s'oriente autour de trois questions : tout d'abord, quelles sont les difficultés spécifiques que rencontrent les pays qui ont dû mettre en œuvre les mesures de GJ en démarrant de zéro ? Quelle comparaison peut-on effectuer entre leurs difficultés et celles qu'ont rencontrées les pays qui adaptent des structures existantes ? Et, enfin, dans quelle mesure l'importance des réformes que les pays membres doivent mettre en place pour répondre aux exigences de la GJ affectet-elle les coûts de mise en œuvre du dispositif ?

\section{BibliograPHIE}

BeCKER G. S. (1994), Human Capital: A Theoretical and Empirical Analysis with Special Reference to Education, Chicago, The University of Chicago Press.

Bellmann L., JaCKMAn R. (1996), “The Impact of Labour Market Policy on Wages, Employment and Labour Market Mismatch", in Schmid G., O'Reilly J., Schömann K. (eds.), International Handbook of Labour Market Policy and Evaluation, Cheltenham, Edward Elgar.

Bussi M., GeYER, L. (2013), Youth Guarantees and Recent Developments on Measures Against Youth Unemployment: A Mapping Exercise, Brussels, European Trade Union Institute. 
Blundell B., Costa Dias M., Meghir C., Van Reenen J. (2004), "Evaluating the Employment Impact of a Mandatory Job Search Program", Journal of the European Economic Association, $\mathrm{n}^{\mathrm{o}} 2$, pp. 569-606.

Bratti M., Ghirelli C., Havari E., Santangelo G. (2017), Vocational Training and Labour Market Outcomes: Evidence from the Youth Guarantee in Latvia, European Commission Joint Research Center Working Paper.

CALIEndo M., KünN S. (2014), "Regional Effect Heterogeneity of Start-up Subsidies for the Unemployed”, Regional Studies, vol. 48, n 6, pp. 1108-1134.

CAlmfors L., LANG H. (1995), "Macroeconomic Effects of Active Labour Market Programmes in a Union Wage-Setting Model”, The Economic Journal, vol. 105, n 430, pp. 601-619.

Calmfors L., Skedinger P. (1995), “Does Active Labour Market Policy Increase Employment? - Theoretical Considerations and some Empirical Evidence from Sweden”, Seminar Papers, $\mathrm{n}^{\mathrm{o}}$ 590, Institute for International Economic Studies, Stockholm, Stockholm University.

Card D., Kluve J., Weber A. (2010), “Active Labor Market Policy Evaluations: A MetaAnalysis”, The Economic Journal, n 120, pp. 452-77.

Card D., Kluve J., Weber A. (2017), “What Works? A Meta- Analysis of Recent Active Labor Market Program Evaluations”, NBER Working Papers Series, $\mathrm{n}^{\circ}$ 21431, Cambridge MA, National Bureau of Economic Research.

Carmeci L., Mauro L. (2003), “Long Run Growth and Investment in Education: Does Unemployment Matter?” Journal of Macroeconomics, vol. 25, n 1, pp. 123-137.

Cockx B., Van Belle E. (2016), Waiting Longer Before Claiming, and Activating Youth: No Point?, Munich, CESifo Working Paper, nº 6104.

DARES (2016), Rapport intermédiaire du Comité scientifique en charge de l'évaluation de la Garantie jeunes, novembre 2016, Paris.

De Giorgi G. (2005), “The New Deal for Young People: Five Years On”, Fiscal Studies, n 26, pp. 371-383.

Dev M. (1995), “India's (Maharashtra) Employment Guarantee Scheme: Lessons from Long Experience", in von Braun J. (ed.), Employment for Poverty Reduction and Food Security, Washington DC, International Food Policy Research Institute, pp. 108-143.

Dolton P., O’Neill D. (1996), “Unemployment Duration and the Restart Effect: Some Experimental Evidence”, Economic Journal, n ${ }^{\circ} 106$, pp. 387-400.

DrÈze J., SEn A. (1989), "Strategies of Entitlement Protection”, in Drèze J., Sen A. (eds.), Hunger and Public Action, Oxford, Oxford University Press, pp. 104-121.

Edgerton J. D., Roberts L. W., Von Below S. (2012), "Education and Quality of Life", in Land K. C., Michalos A. C., Sirgy J. (eds.), Handbook of Social Indicators and Quality of Life Research, Springer.

Escudero V. (2015), "Are Active Labour Market Policies Effective in Activating and Integrating Low-Skilled Individuals? An International Comparison”, Research Department Working Paper, $\mathrm{n}^{\mathrm{o}} 3$, Geneva, International Labour Office. 
Escudero V., LóPez Mourelo E. (2015), “The Youth Guarantee Programme in Europe: Features, Implementation and Challenges", Research Department Working Paper, $\mathrm{n}^{\circ} 4$, Geneva, International Labour Office.

Escudero V., Khün S., LóPez Mourelo E., Tobin S. (2017), “Youth Labour Market Prospects and Decent Policy Developments", in Malo M. A., Moreno A. (eds.), European Youth Labour Markets: Problems and Polices, Springer.

EuRopean COMmission (EC) (2003), Green paper: Entrepreneurship in Europe, 27 final, January, Brussels, European Commission.

European COMmission (EC) (2010), Youth on the Move: An Initiative to Unleash the Potential of Young People to Achieve Smart, Sustainable and Inclusive Growth in the European Union, Communication from the Commission to the European Parliament, the Council, the European Economic and Social Committee and the Committee of the Regions, 477 final, September, Brussels, European Commission.

European COMMISSION (EC) (2012a), Towards a Job-Rich Recovery, Communication from the Commission to the European Parliament, the Council, the European Economic and Social Committee and the Committee of the Regions, 173 final, Brussels, European Commission.

EuRoPEAN COMMISSION (EC) (2012b), Proposal for a Council Recommendation on Establishing a Youth Guarantee, 729 final, December, Brussels, European Commission.

EuROPEAN COMMISSION (EC) (2012c), Commission StaffWorking Document accompanying the document Proposal for a Council Recommendation on Establishing a Youth Guarantee, 409 final, December, Brussels, European Commission.

European Commission (EC) (2013), Conclusions of the European Council of 27/28 June, EUCO 104/2/13, June, Brussels, European Council.

EUROPEAN COMMISSION (EC) (2016a), The Youth Guarantee and Youth Employment Initiative Three Years on, Communication from the Commission to the European Parliament, the European Council, the European Economic and Social Committee and the Committee of the Regions, SWD (2016) 323 final, October, Brussels, European Commission.

EUROPEAN COMMISSION (EC) (2016b), The Youth Guarantee and Youth Employment Initiative Three Years on, Staff working document accompanying the document "Communication from the Commission to the European Parliament, the European Council, the European Economic and Social Committee and the Committee of the Regions", SWD (2016) 323 final, October, Brussels, European Commission.

EUROPEAN COMMISSION (EC) (2016c), First Results of the Youth Employment Initiative, A Final Report to DG Employment, Social Affairs and Inclusion of the European Commission, June, Brussels, European Commission.

European Commission (EC) (2016d), Mid-Term Review/Revision of the Multiannual Financial Framework 2014-2020. An EU Budget Focused on Results, Communication from the Commission to the European Parliament and the Council, SWD (2016) 299 final, September, Brussels, European Commission. 
European COUnCIL (2013), Council Recommendation of 22 April 2013 on Establishing a Youth Guarantee, 2013/C 120/01, April, Brussels, European Council.

Farvaque N., Kramme C., Tuchszirer C. (2016), « La Garantie jeunes du point de vue des missions locales : un modèle d'accompagnement innovant, mais source de bouleversements organisationnels », Rapport de recherche, $\mathrm{n}^{\circ}$ 102, Paris, Centre d'études de l'emploi et du travail.

Graversen K. B., van Ours J. C. (2008), "How to Help Unemployed Find Jobs Quickly: Experimental Evidence from a Mandatory Activation Program", Journal of Public Economics, vol. 92, pp. 2020-2035.

HÄMÄLÄINEN K., HÄMÄLÄINEN U., TUOMALA J. (2017), “The Labour Market Impacts of a Youth Guarantee: Lessons for Europe?”, in Malo M. A., Moreno A. (eds.), European Youth Labour Markets: Problems and Polices, Springer, Chapter 5.

Hardoy I. (2005), "Impact of Multiple Labour Market Programmes on Multiple Outcomes: The Case of Norwegian Youth Programmes", Labour, vol. 19, n 3, pp. 425-467.

International Labour Organization (ILo) (2012), Eurozone Job Crisis: Trends and Policy responses. Studies on Growth with Equity, Geneva, International Institute for Labour Studies.

INTERNATIONAL LABOUR ORGANIZATION (ILO) (2016), What works. Active labour market policies in Latin America and the Caribbean, Geneva, International Labour Organization.

Jensen P., Rosholm M., Svarer M. (2003), "The Response of Youth Unemployment to Benefits, Incentives and Sanctions", European Journal of Political Economy, vol. 19, n ${ }^{\circ}$ 2, pp. 301-316.

LAYARD R. (1996), "Preventing Long Term Unemployment: An Economic Analysis", in Snower D., De La Dehesa G. (eds.), Unemployment Policy: Government Options for the Labour Market, Cambridge, Cambridge University Press.

Layard R., Nickell S., JackMAn R. (2009), Unemployment: Macroeconomic Performance and the Labour Market, Oxford, Oxford University Press.

Loison-Leruste M., Couronné J., SARfati F. (2016), « La Garantie jeunes en action : usages du dispositif et parcours de jeunes », Rapport de recherche, $\mathrm{n}^{\circ} 101$, Paris, Centre d'études de l'emploi et du travail.

MAscherini M. (2012), Youth guarantee: Experiences from Finland and Sweden, Dublin, European Foundation for the Improvement of Living and Working Conditions.

Mascherini M., Ludwinek A., Vacas C., Meierkord A., Gebel M. (2014), Mapping Youth Transitions in Europe, Dublin, European Foundation for the Improvement of Living and Working Conditions.

Micklewright J., NAGy G. (2010), “The Effect of Monitoring Unemployment Insurance recipients on Unemployment Duration: Evidence from a Field experiment", Labour Economics, $\mathrm{n}^{\mathrm{o}} 17$, pp. 180-187.

MinCER J. (1974), Schooling, experience and earnings, New York, National Bureau of Economic Research. 
Organisation for EConomic Co-operation and Development (OecD) (1993), "Active Labour Market Policies: Assessing Macroeconomic and Microeconomic Effects", in OECD (ed.), Employment Outlook, Chapter 2, Paris, OECD, pp. 39-80.

ORgANisATION FOR ECONOMiC Co-OPERATION AND DEVELOPMENT (OECD) (2015), The Local Implementation of Youth Guarantees: Emerging Lessons from European Experiences, Paris, OECD.

PISSARIDES C.A. (1990), Equilibrium Unemployment Theory, Cambridge MA, The MIT Press.

Ravallion M. (1991), "Reaching the Rural Poor Through Public Employment”, The World Bank Research Observer, vol. 6, n 2, pp. 153-175.

Schmid G. (1996), "New Public Management of Further Training”, in Schmid G., O'Reilly J., Schömann K. (eds.), International Handbook of Labour Market Policy and Evaluation, Cheltenham, Edward Elgar, pp. 725-746.

Schmid G., Speckesser S., Hilbert C. (2001), "Does Active Labour Market Policy Matter? An Aggregate Impact Analysis for Germany", in de Koning J., Mosley H. (eds.), Labour Market Policy and Unemployment: Impact and Process Evaluations in Selected European Countries, Cheltenham, Edward Elgar, pp. 78-114.

Schultz T. (1975), "The Value of the Ability to Deal With Disequilibria", Journal of Economic Literature, vol. 13, $\mathrm{n}^{\mathrm{o}} 3$, pp. 827-846.

Thurow L. (1975). Generating Inequality: Mechanisms of Distribution in the U.S. Economy, New York, Basic Books.

VAN DEN Berg G. J., van DER KlaAuw B. (2006), "Counseling and Monitoring of Unemployed Workers: Theory and Evidence from a Controlled Social Experiment", International Economic Review, vol. 47, $\mathrm{n}^{\circ} 3$ pp. 895-936.

Van den Berg G. J., Bergemann A. H., Caliendo M. (2009), "The Effect of Active Labor Market Programs on Not-Yet Treated Unemployed Individuals", Journal of the European Economic Association, n ${ }^{\circ}$ 7, pp. 606-616. 


\section{ANNEXe 1 - Liste DeS ABRÉVIATIONS}

CE : Commission européenne

Dares : Direction de l'animation de la recherche, des études et des statistiques

GJ : Garantie jeunesse

IEJ : Initiative pour l'emploi des jeunes

Neet : Neither in Employment nor in Education or Training (ni en emploi, ni en études ou formation)

OCDE : Organisation de coopération et de développement économiques

OIT : Organisation internationale du travail

PAMT : Politiques actives du marché du travail

PIB : Produit intérieur brut

PPA : Parité de pouvoir d'achat

SPE : Service public de l'emploi

UE-28 : Union européenne à 28 pays membres 
ANNEXE 2 - INDICATEURS CONCERNANT LE MARCHÉ DU TRAVAIL POUR LES JEUNES (15-24 ANS) PAR PAYS (2013-2016)

\begin{tabular}{|c|c|c|c|c|c|c|}
\hline & \multicolumn{2}{|c|}{ Taux de chômage } & \multicolumn{2}{|c|}{ Taux d'activité } & \multicolumn{2}{|c|}{ Taux de Neet } \\
\hline & 2013 & 2016 & 2013 & 2016 & 2013 & 2016 \\
\hline UE-28 & 23,6 & 18,7 & 32,1 & 33,7 & 13,0 & 11,5 \\
\hline Allemagne & 7,8 & 7,1 & 46,9 & 45,7 & 6,3 & 6,6 \\
\hline Autriche & 9,7 & 11,2 & 53,1 & 51 & 7,3 & 7,7 \\
\hline Belgique & 23,7 & 20,1 & 23,6 & 22,7 & 12,7 & 9,9 \\
\hline Bulgarie & 28,4 & 17,2 & 21,2 & 19,8 & 21,6 & 18,2 \\
\hline Croatie & 50,0 & 31,3 & 14,9 & 25,6 & 19,6 & 16,9 \\
\hline Chypre & 38,9 & 29,1 & 23,5 & 26,4 & 18,7 & 15,9 \\
\hline Danemark & 13,1 & 12,0 & 53,7 & 58,2 & 6,0 & 5,8 \\
\hline Espagne & 55,5 & 44,4 & 16,8 & 18,4 & 18,6 & 14,6 \\
\hline Estonie & 18,7 & 13,4 & 32,4 & 37,5 & 11,3 & 9,1 \\
\hline Finlande & 19,9 & 20,1 & 41,5 & 41,7 & 9,3 & 9,9 \\
\hline France & 24,1 & 24,6 & 28,4 & 27,8 & 11,2 & 11,9 \\
\hline Grèce & 58,3 & 47,3 & 11,8 & 13,0 & 20,4 & 15,8 \\
\hline Hongrie & 26,6 & 12,9 & 20,1 & 28,1 & 15,5 & 11,0 \\
\hline Irlande & 26,8 & 17,2 & 29,0 & 32,1 & 16,1 & 13,0 \\
\hline Italie & 40,0 & 37,8 & 16,3 & 16,6 & 22,2 & 19,9 \\
\hline Lettonie & 23,2 & 17,3 & 30,2 & 32,8 & 13,0 & 11,2 \\
\hline Lituanie & 21,9 & 14,5 & 24,6 & 30,2 & 11,1 & 9,4 \\
\hline Luxembourg & 15,5 & 18,9 & 21,9 & 24,9 & 5,0 & 5,4 \\
\hline Malte & 13,0 & 11,0 & 46,0 & 46,2 & 9,9 & 8,6 \\
\hline Pays-Bas & 13,2 & 10,8 & 60,1 & 60,8 & 5,6 & 4,6 \\
\hline Pologne & 27,3 & 17,7 & 24,2 & 28,4 & 12,2 & 10,5 \\
\hline Portugal & 38,1 & 28,0 & 21,7 & 23,9 & 14,1 & 10,6 \\
\hline République tchèque & 19,0 & 10,5 & 25,6 & 28,6 & 9,1 & 7,0 \\
\hline Roumanie & 23,7 & 20,6 & 22,9 & 22,3 & 17,0 & 17,4 \\
\hline Royaume-Uni & 20,7 & 13 & 46,3 & 50,8 & 13,2 & 10,9 \\
\hline Slovaquie & 33,7 & 22,2 & 20,4 & 25,2 & 13,7 & 12,3 \\
\hline Slovénie & 21,6 & 15,2 & 26,5 & 28,6 & 9,2 & 8,0 \\
\hline Suède & 23,5 & 18,9 & 41,7 & 44,5 & 7,5 & 6,5 \\
\hline
\end{tabular}




\section{Annexe 3 - Chronologie de la mise en deuvre de la Garantie JEUNESSE EUROPÉENNE}

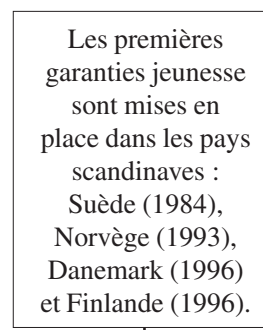

$1980-$ 1990
Des discussions à propos d'une initiative sur la garantie jeunesse sont menées avec les partenaires sociaux.
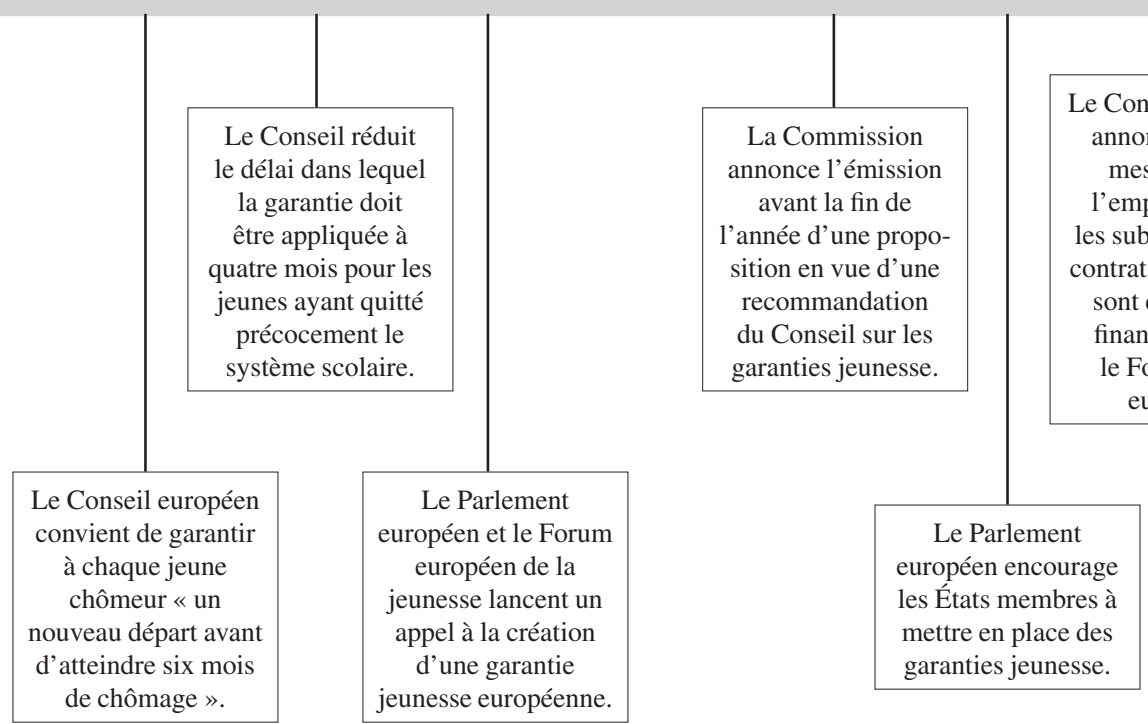
Les partenaires sociaux discutent une nouvelle fois de la possibilité d'une initiative sur la garantie jeunesse.

\section{Octobre Décembre Février Avril Juin Mai 2014- Septembre 2012 2012

Le Conseil européen convient de lancer l'Initiative pour l'emploi des jeunes (IEJ) avec un fonds de 6,4 milliards d'euros pour soutenir l'ensemble des mesures comprises dans le Paquet en faveur de l'emploi des jeunes de la Commission, et en particulier la GJ.
Le Conseil européen statue que les 6,4 milliards d'euros seront payés dès 2014-2016 et que les pays bénéficiaires devront soumettre un plan de mise en œuvre de la Garantie jeunesse avant la fin de l'année.
La mise en œuvre des programmes nationaux de la Garantie jeunesse est lancée.
La Commission lance le Paquet en faveur de l'emploi des jeunes et formule une proposition pour une recommandation du Conseil sur la mise en place d'une garantie jeunesse
Le Conseil adopte la recommandation sur la mise en place de la GJ.
Tous les États membres soumettent leurs plans de mise en œuvre.
La Commission propose d'augmenter le budget de l'IEJ de 2,2 milliards pour la période 2017-2020. 
